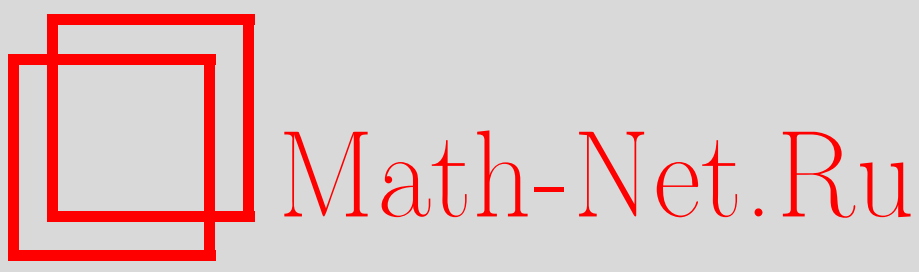

Г. Г. Магарил-Ильяев, В. М. Тихомиров, О неравенствах для производных колмогоровского типа, Матем. сб., 1997, том 188, номер 12, 73-106

DOI: https://doi.org/10.4213/sm274

Использование Общероссийского математического портала Math-Net.Ru подразумевает, что вы прочитали и согласны с пользовательским соглашением

http://www . mathnet.ru/rus/agreement

Параметры загрузки:

IP : 35.173 .219 .149

26 апреля 2023 г., 12:19:55 


\section{О неравенствах для производных колмогоровского типа}

Общая задача о неравенствах для производных состоит в оценке нормы некоторой производной функции через нормы других ее производных. Цель работырассмотрение подобных задач с общих позиций теории экстремума.

Библиографйл: 26 названий.

\section{Введение}

Под неравенствами для производных колмогоровского типа традиционно понимают мультипликативные неравенства вида

$$
\left\|x^{(k)}(\cdot)\right\|_{L_{q}(T)} \leqslant K\|x(\cdot)\|_{L_{p}(T)}^{\alpha}\left\|x^{(n)}(\cdot)\right\|_{L_{r}(T)}^{\beta}
$$

(где $0 \leqslant k<n$ - целые, $1 \leqslant p, q, r \leqslant \infty, \alpha, \beta \geqslant 0, T=\mathbb{R}$ или $\mathbb{R}_{+}$), справедливые для всех функций $x(\cdot) \in L_{p}(T)$, у которых $(n-1)$-я производная локально абсолютно непрерывна на $T$ и $x^{(n)}(\cdot) \in L_{r}(T)$. Пространство таких функций будем обозначать через $\mathscr{W}_{p, r}^{n}(T)$ или просто $\mathscr{W}_{p}^{n}(T)$, если $p=r$.

При фиксированном $T$ неравенство (1) зависит от пяти параметров: $n, k, p, q$ и $r$ (величины $\alpha$ и $\beta$ однозначно ими определяются: $\alpha=(n-k-1 / r+1 / q) \times$ $\left.(n-1 / r+1 / p)^{-1}, \beta=1-\alpha\right)$. Точную (т.е. наименшшую возможную) константу в этом неравенстве будем обозначать через $K_{T}(n, k, p, q, r)$.

Первые точные неравенства типа (1) были получены в работах Ландау [1] $\left(T=\mathbb{R}_{+}, n=2, k=1, p=q=r=\infty\right)$ и Адамара [2] $(T=\mathbb{R}, n=2, k=1$, $p=q=r=\infty)$. В конце тридцатых годов в работе Колмогорова [3] были найдены точные константы в (1) при $T=\mathbb{R}, p=q=r=\infty$ в общем случае, т.е. при любых $n \geqslant 2$ и $0<k<n$. Этот результат и поныне остается одним из наиболее ярких в данной проблематике, и точные неравенства вида (1) нередко назьвают неравенствами Колмогорова.

Значительные усилия были затрачены на нахождение точных констант в подобных неравенствах при различных значениях параметров, этой тематике посвящены десятки работ. Но результаты, аналогичные по своей завершенности колмогоровскому, получены лишь еще в пяти случаях: в трех случаях на $\mathbb{R}(p=q=r=2-$ Харди-Литтлвуд-Полиа [4], $p=q=r=1$ - Стейн [5], $p=r=2, q=\infty$ - Тайков [6]) и в двух случаях на $\mathbb{R}_{+}(p=q=r=2$ - Любич [7], Кущцов [8] и $p=r=2$, $q=\infty-$ Габушин [9]).

Работа выполнена при финансовой поддержке Российского фонда фундаментальных исследований (грант № 96-01-00325) и программы поддержки ведущих научных школ РФ (грант № 96-15-96072). 
Кроме названных "общих" решений, имеется несколько десятков "частных", т.е. когда точные константы найдены при каких-то фиксированных значениях параметров $n, k, p, q$ и $r$. Большинство частных решений относится к случаю $n=2$, $k=0,1$, и если значения других параметров изображать точками единичного куба: $0 \leqslant 1 / p, 1 / q, 1 / r \leqslant 1$, то они расположены в двух плоскостях: $1 / q=0$ и $(2-r) / r+k / p=2 / q$. Перечень найденных точных констант в неравенстве (1) см. в статьях Тихомирова, Магарил-Ильяева [10] и Арестова [11].

Задача о вычислении точной константы в неравенстве (1) равносильна нахождению точного значения следуюшей экстремальной задачи:

$$
\left\|x^{(k)}(\cdot)\right\|_{L_{q}(T)} \rightarrow \max , \quad\|x(\cdot)\|_{L_{p}(T)} \leqslant \gamma_{1}, \quad\left\|x^{(n)}(\cdot)\right\|_{L_{r}(T)} \leqslant \gamma_{2}
$$

для любых фиксированных чисел $\gamma_{1}>0$ и $\gamma_{2}>0$.

В этой работе, трактуя именно так задачу о неравенствах для производных, мы будем рассматривать и более общие постановки:

$$
\left\|D_{0} x(\cdot)\right\|_{L_{p_{0}}(T)} \rightarrow \max , \quad\left\|D_{j} x(\cdot)\right\|_{L_{p_{j}}(T)} \leqslant \gamma_{j}, \quad j=1, \ldots, N,
$$

где $T$ - некоторое многообразие (прямая, полупрямая, отрезок, сфера, пространство $\mathbb{R}^{d}$ и др.) и $D_{j}, j=0,1, \ldots, N,-$ некоторые дифференциальные операторы.

В данной работе мы исследуем подобные обобщения задач о неравенствах для производных, соответствуюших названным выше пяти типам. Частным решениям будет посвящена отдельная публикация.

Задачи (2) и (3) относятся к так называемым экстремальным задачам, т.е. к задачам на максимум и минимум. При решении подобных задач мы, как правило, будем поступать единообразно, придерживаясь следуюшей схемы:

1) формализация (т.е. запись задачи в виде минимизации или максимизации некоторого функционала при ограничениях типа равенств, неравенств и включений);

2) применение принципа Лагранжа или методов двойственности (т.е. формулировка необходимых условий экстремума или выписьвание двойственных соотношений, которые приводят к некоторым уравнениям и/или неравенствам);

3) исследование полученных уравнений и/или неравенств (т.е. нахождение их решений и применение достаточных условий экстремума);

4) формулировка окончательного результата.

То, что мы мы назьваем принципом Лагранжа, сам Лагранж сформулировал как необходимое условие экстремума в задачах с ограничениями, которые задаются равенствами. Несколько модифицировав основную идею Лагранжа, распространив ее и на задачи, ограничения в которых (помимо равенств) могут задаваться неравенствами и включениями, можно сказать, что замысел Лагранжа заключается в следуюшем: для получения необходимых условий әкстремума в такой задаче надо составить функиию (функиию Лагранжа), которая есть сумма минимизируемого (или максимизируемого) функиионала и функиий, задающих равенства и неравенства с неопределенными множителями (множителями Лагранжа), а затем выписать необходимые условия әкстремума в более 
простой задаче - в задаче на әкстремум функиии Лагранжа “как если бъ переменные были независимы" (в кавычках приведены подлинные слова Лагранжа), если ограничений типа включений нет, а если они есть, то выписать необходимые условия с учетом әтих ограничений.

Идея Лагранжа обладает значительной универсальностью и лежит в основе многих необходимых условий экстремума, встречающихся в анализе (начиная с правила множителей Лагранжа, сформулированного впервые в середине XVIII века и кончая принципом максимума для задач с распределенными параметрами, доказанным сравнительно недавно). Тематике, связанной с принципом Лагранжа, один из авторов посвятил ряд работ (упомянем лишш книги [12]-[17]). Но, как правило, мы не будем пользоваться доказанньми результатами на эту тему. Мы будем применять принцип Лагранжа или методы двойственности как әвристические nриемы (т.е. не заботясь о том, выполнены или не выполнены те или иные условия их применения) и, решив полученные в результате этой процедуры уравнения и/или неравенства, будем доказывать (используя различные достаточные условия), что на самом деле мы нашли решение поставленной задачи. Заметим при этом, что проверка достаточности (особенно в выпуклых задачах) зачастую почти тривиальна.

Ниже мы формулируем принцип Лагранжа для достаточно широкого класса экстремальных задач и считаем, не ограничивая общности, что это задачи минимизации. Начнем с задачи математического программирования:

$$
f_{0}(x) \rightarrow \min , \quad f_{i}(x) \leqslant 0,1 \leqslant i \leqslant m, \quad f_{i}(x)=0, m+1 \leqslant i \leqslant N .
$$

Здесь $f_{i}: X \rightarrow \mathbb{R} \cup\{+\infty\}, 0 \leqslant i \leqslant N, X$ - линейное (обычно нормированное) пространство. Функция Лагранжа имеет вид:

$$
\mathscr{L}(x, \lambda)=\sum_{i=0}^{N} \lambda_{i} f_{i}(x)
$$

где $\lambda=\left(\lambda_{0}, \lambda_{1}, \ldots, \lambda_{N}\right)$ и числа $\lambda_{i}, 0 \leqslant i \leqslant N$, называются множсителями Лагранәса. Принцип Лагранжа для данной задачи заключается в том, что должны найтись такие $\lambda_{i}, i=0,1, \ldots, N$ (причем $\lambda_{i} \geqslant 0, i=0,1, \ldots, m$ ), не все равные нулю, что решение $(\mathrm{P})$ удовлетворяет необходимым условиям экстремума в задаче: $\mathscr{L}(x, \lambda) \rightarrow \min , x \in X$. Если все функции $f_{i}, 0 \leqslant i \leqslant N$, гладкие, то, очевидно, это приводит к уравнению: $\partial \mathscr{L}(x, \lambda) / \partial x=0$. При этом выполняются еще условия дополняющей нежесткости: $\lambda_{i} f_{i}(x)=0,1 \leqslant i \leqslant m$.

Если в задаче $(\mathrm{P})$ функционалы таковы, что

$$
f_{i}(x(\cdot), u(\cdot))=\int_{t_{0}}^{t_{1}} g_{i}(t, x(t), u(t)) d t+h_{i}\left(x\left(t_{0}\right), x\left(t_{1}\right)\right), \quad i=0,1, \ldots, N,
$$

и кроме того, $\dot{x}=\varphi(t, x, u)$, где $x(\cdot)$ и $u(\cdot)$ - вектор-функции со значениями в $\mathbb{R}^{n}$ и $\mathbb{R}^{r}$, соответственно, то мы получаем так называемую задачу Лагранжса, которая относится к классическому вариачионному исчислению. Функция Лагранжа имеет вид:

$$
\mathscr{L}=\int_{t_{0}}^{t_{1}} L d t+l, \quad L=\sum_{i=0}^{N} \lambda_{i} g_{i}+\langle p, \dot{x}-\varphi\rangle, \quad l=\sum_{i=0}^{N} \lambda_{i} h_{i}
$$


(слагаемое $\langle p, \dot{x}-\varphi\rangle$, где $\langle\cdot, \cdot\rangle$ - скалярное произведение в $\mathbb{R}^{n}$ и $p$-вектор-фуункция, появляется здесь вследствие того, что для каждого $t \in\left[t_{0}, t_{1}\right]$ соотношение $\dot{x}=\varphi(t, x, u)$ рассматривается как ограничение, задаваемое $n$ равенствами).

В соответствии с принципом Лагранжа мы должны выписать необходимые условия экстремума в задаче без ограничений: $\int_{t_{0}}^{t_{1}} L d t+l \rightarrow \min$. Они состоят из двух частей: уравнения Эйлера

$$
-\frac{d}{d t} L_{\dot{x}}+L_{x}=0, \quad L_{u}=0
$$

и условия трансверсальности

$$
L_{\dot{x}}\left(t_{i}, x\left(t_{i}\right), \dot{x}\left(t_{i}\right)\right)=(-1)^{i} l_{x\left(t_{i}\right)}\left(x\left(t_{i}\right), \dot{x}\left(t_{i}\right)\right), \quad i=0,1
$$

Если в дополнение к ограничениям в задаче Лагранжа наложено условие $u(t) \in U$ для почти всех $t$, где $U-$ некоторое подмножество в $\mathbb{R}^{r}$ (такие задачи называют задачами оптимального управления), то вместо уравнения $L_{u}=0$ выполняется (для почти всех $t$ ) приниип минимума:

$$
\min _{u \in U} L(t, x(t), \dot{x}(t), u)=L(t, x(t), \dot{x}(t), u(t))
$$

Отметим также, что если в задаче есть еще и фазовые ограничения типа неравенств, т.е. ограничения вида $G(t, x(t)) \leqslant 0$, то в функцию Лагранжа добавляется слагаемое $\int_{t_{0}}^{t_{1}} G d \mu(t)(d \mu(\cdot)$ - неотрицательная мера, сосредоточенная там, где неравенства обращаются в равенства), смысл которого в том, что фазовые ограничения рассматриваются как континуум ограничений типа неравенств (впрочем, как и дифференциальная связь - это континуум равенств).

Среди конкретных задач, с которьми мы будем иметь дело, большинство окажутся выпукльми. Напомним, что функция $f: X \rightarrow \overline{\mathbb{R}}:=\mathbb{R} \cup\{-\infty\} \cup\{+\infty\}$, где $X$ - вешественное линейное пространство, назьвается вылуклой, если ее надграфик epi $f:=\{(x, \alpha) \in X \times \mathbb{R}: \alpha \geqslant f(x)\}$ есть выпуклое подмножество в $X \times \mathbb{R}$.

Пусть в задаче $(\mathrm{P}) \quad X$ - вешественное линейное пространство, функции $f_{i}$, $0 \leqslant i \leqslant m,-$ выпуклы, функции $f_{i}, m+1 \leqslant i \leqslant N,-$ аффинны (сумма линейной и постоянной функций) и есть еще ограничение типа включения: $x \in A$, где $A$ - выпуклое подмножество $X$. В этом случае $(\mathrm{P})$ называется задачей выпуклого программирования. Для таких задач принцип Лагранжа имеет завершенную форму, что находит выражение в следуюшем результате.

ТЕОРЕМА КУНА-ТАККЕРА. Пусть $\widehat{x}$ - абсолютный минимум в указанной задаче выпуклого программирования. Тогда найдутся множители Лагранжа $а \lambda_{i}, i=0,1, \ldots, N$, не равнье нулю одновременно, такие, что

(a) $\lambda_{i} \geqslant 0$ для $0 \leqslant i \leqslant m$;

(b) выполнень условия дополняющей нежесткости: $\lambda_{i} f\left(\widehat{x}_{i}\right)=0,1 \leqslant i \leqslant m$;

(c) $\widehat{x}$ есть абсолютный минимум в задаче: $\mathscr{L}(x, \lambda):=\sum_{i=0}^{N} \lambda_{i} f_{i}(x) \rightarrow \min$, $x \in A$. 
Если для некоторой допустимой точки $\widehat{x}$ выполнены условия (a), (b), (c) с $\lambda_{0} \neq 0$, то $\widehat{x}-$ абсолютный минимум в задаче.

Легко проверить, что если в задаче вьпуклого программирования, где отсутствуют ограничения типа равенств, найдется такое $\bar{x} \in A$, что $f_{i}(\bar{x})<0, i \neq 0$ (условие Слейтера), то $\lambda_{0} \neq 0$.

Кроме принципа Лагранжа мы будем пользоваться, как уже было отмечено, методами двойственности для выпуклых задач. Двойственность - это возможность двойного описания выпуклых объектов (множеств, функций, экстремальных задач). Одно описание - непосредственное, т.е. в терминах исходного пространства, а другое - в терминах двойственного пространства.

Двойственность выпуклых функций описывается с помощью понятия сопряженной функции. Пусть $X$ и $X^{*}$ - два вешественных линейных пространства, находящиеся в двойственности относительно билинейной формы $\langle\cdot, \cdot\rangle: X^{*} \times X \rightarrow \mathbb{R}$ (обычно $X^{*}$ - сопряженное к $X$ (алгебраически или топологически, если, скажем, $X$ - нормировано) и $\left\langle x^{*}, x\right\rangle$ - значение линейного функционала $x^{*} \in X^{*}$ на элементе $x \in X)$, и $f: X \rightarrow \overline{\mathbb{R}}$ - некоторая функция (не обязательно выпуклая). Функция $f^{*}: X^{*} \rightarrow \overline{\mathbb{R}}$, определенная равенством

$$
f^{*}\left(x^{*}\right):=\sup _{x \in X}\left(\left\langle x^{*}, x\right\rangle-f(x)\right),
$$

называется сопряженной $\kappa f$ (относительно данной двойственности) или преобразованием Юнга, или Юнга-Фенхеля, или Лежсандра.

Поскольку $f^{*}$ есть верхняя грань афффинных функций $x^{*} \mapsto\left\langle x^{*}, x\right\rangle-f(x)$, то $f^{*}$ - вьпуклая функция.

Второй сопряжснной $\kappa f$ называется функция, задаваемая равенством

$$
f^{* *}(x):=\sup _{x^{*} \in X^{*}}\left(\left\langle x^{*}, x\right\rangle-f^{*}\left(x^{*}\right)\right) .
$$

Это также выпуклая функция и легко проверить, что $f(x) \geqslant f^{* *}(x)$ для всех $x \in X$.

Точная расшифровка понятия двойственности выпуклых функций содержится в следующей теореме, лежащей в основе многих утверждений, связанных с двойственностью выпуклых задач.

ТЕОРема ФЕНХЕЛЯ-МОРО. Пусть $X$ и $X^{*}$ - вещественные линейные пространства в двойственности $u f: X \rightarrow \mathbb{R} \cup\{+\infty\}$. Тогда $f^{* *}=f$ в том $и$ только в том случае, когда $f$ выпукла и замкнута (т.е. множсество ері $f$ замкнуто в слабой топологии $\sigma\left(X, X^{*}\right)$, порожсденной двойственностью $X$ $\left.u X^{*}\right)$.

Таким образом, эта теорема утверждает, что выпуклая замкнутая функция, определяемая, с одной стороны, своим надграфиком, является и верхней гранью семейства непрерывных (в топологии $\sigma\left(X, X^{*}\right)$ ) афффинных функций $x \mapsto\left\langle x^{*}, x\right\rangle-f^{*}\left(x^{*}\right), x^{*} \in X^{*}$.

Приведем теперь общую схему построения задачи двойственной к данной. Пусть $X$ и $X^{*}$ - вещественные линейные пространства в двойственности относительно билинейной формы $\langle\cdot, \cdot\rangle_{1}$ и $f: X \rightarrow \overline{\mathbb{R}}$. Рассмотрим задачу

$$
f(x) \rightarrow \min , \quad x \in X .
$$


Пусть, далее, $Y$ и $Y^{*}$ - другая пара пространств в двойственности относительно билинейной формы $\langle\cdot, \cdot\rangle_{2}$ и функция $F: X \times Y \rightarrow \overline{\mathbb{R}}$ такова, что $F(x, 0)=f(x)$ для всех $x \in X$. Каждому $y \in Y$ сопоставим задачу

$$
F(x, y) \rightarrow \min , \quad x \in X
$$

Семейство таких задач называется возмущением задачи (Я). Двойственной задачей $\kappa(\mathscr{P})$ (относительно заданного возмущения) называется задача

$$
-F^{*}\left(0, y^{*}\right) \rightarrow \max , \quad y^{*} \in Y^{*}
$$

где $F^{*}: X^{*} \times Y^{*} \rightarrow \overline{\mathbb{R}}-$ сопряженная функция к $F$ (относительно естественной двойственности между $X \times Y$ и $\left.X^{*} \times Y^{*}\right)$.

В основе этой схемы лежит все та же двойственность выпуклых функций. Действительно, если $S(y)$ - значение задачи $\left(\mathscr{P}_{y}\right)$, тогда согласно предыдущему $S(0) \geqslant S^{* *}(0)=\sup _{y^{*} \in Y^{*}}\left(-S^{*}\left(y^{*}\right)\right)$. По определению

$$
\begin{aligned}
S^{*}\left(y^{*}\right) & =\sup _{y \in Y}\left(\left\langle y^{*}, y\right\rangle_{2}-\inf _{x \in X} F(x, y)\right) \\
& =\sup _{x \in X, y \in Y}\left(\left\langle x^{*}, 0\right\rangle_{1}+\left\langle y^{*}, y\right\rangle_{2}-F(x, y)\right)=F^{*}\left(0, y^{*}\right)
\end{aligned}
$$

Тем самым, очевидна связь задач $(\mathscr{P})$ и $\left(\mathscr{P}^{*}\right)$ и понятно, что условия совпадения их значений могут быть получены из теоремы Фенхеля-Моро. Из приведенных рассуждений вытекает, что значение двойственной задачи не превосходит значения исходной.

В качестве первого примера, которьй понадобится нам ниже, рассмотрим общую задачу линейного программирования. Пусть, по-прежнему, $X$ и $X^{*}$ - вещественные линейные пространства в двойственности относительно билинейной формы $\langle\cdot, \cdot\rangle, x_{j}^{*} \in X^{*}, j=0,1, \ldots, N, \beta_{j} \in \mathbb{R}, j=1, \ldots, N$, и $K$-выпукльй конус в $X$. Задача

$$
\left\langle x_{0}^{*}, x\right\rangle \rightarrow \min , \quad\left\langle x_{j}^{*}, x\right\rangle \leqslant \beta_{j}, \quad j=1, \ldots, N, \quad x \in K,
$$

называется задачей линейного программирования.

Простой подсчет показывает, что двойственная задача к (LP) относительно возмушения

$$
\left\langle x_{0}^{*}, x\right\rangle \rightarrow \min , \quad\left\langle x_{j}^{*}, x\right\rangle \leqslant \beta_{j}+y_{j}, \quad j=1, \ldots, N, \quad x \in K,
$$

где $y_{j} \in \mathbb{R}, j=1, \ldots, N$, имеет вид $\left(\lambda=\left(\lambda_{1}, \ldots, \lambda_{N}\right)\right)$ :

$$
\sum_{j=1}^{N} \lambda_{j} \beta_{j} \rightarrow \max , \quad \lambda \leqslant 0, \quad x_{0}^{*}-\sum_{j=1}^{N} \lambda_{j} x_{j}^{*} \in K^{*}
$$

где $K^{*}=\left\{x^{*} \in X^{*}:\left\langle x^{*}, x\right\rangle \geqslant 0 \forall x \in X\right\}$ - сопряженный конус к $K$.

В качестве второго примера кратко рассмотрим взаимосвязь между задачей о неравенствах для производных и задачей об оптимальном восстановлении 
функционалов. Как уже было отмечено, задача о нахождении точной константы в неравенстве (1) равносильна задаче (2), которая, в свою очередь, при $q=\infty$ имеет, очевидно, то же значение, что и задача

$$
x^{(k)}(0) \rightarrow \max , \quad\|x(\cdot)\|_{L_{p}(T)} \leqslant \gamma_{1}, \quad\left\|x^{(n)}(\cdot)\right\|_{L_{r}(T)} \leqslant \gamma_{2} .
$$

Двойственная задача к (А) относительно возмушения

$$
x^{(k)}(0) \rightarrow \max , \quad\|x(\cdot)+y(\cdot)\|_{L_{p}(T)} \leqslant \gamma_{1}, \quad\left\|x^{(n)}(\cdot)\right\|_{L_{r}(T)} \leqslant \gamma_{2},
$$

как нетрудно проверить, имеет вид:

$$
\begin{aligned}
& \sup _{\|x(\cdot)-y(\cdot)\|_{L_{p(T)} \leqslant \gamma_{1}}}\left(x^{(k)}(0)-\left\langle y^{*}, y(\cdot)\right\rangle\right) \rightarrow \min , \quad y^{*} \in L_{p^{*}}(T), \\
& \left\|x^{(n)}(\cdot)\right\|_{L_{r}(T)} \leqslant \gamma_{2}
\end{aligned}
$$

где $p^{*}=p^{\prime}\left(p^{\prime}-\right.$ сопряженньй показатель к $\left.p\right)$, если $1 \leqslant p<\infty$, а при $p=\infty$ $L_{p^{*}}(T)$ обозначает здесь двойственное пространство к $C^{b}(T)$.

Данную задачу можно трактовать как задачу об оптимальном восстановлении функционала $x(\cdot) \mapsto x^{(k)}(0)$ на классе

$$
W_{p, r}^{n}\left(T, \gamma_{2}\right)=\left\{x(\cdot) \in \mathscr{W}_{p, r}^{n}(T):\left\|x^{(n)}(\cdot)\right\|_{L_{r}(T)} \leqslant \gamma_{2}\right\}
$$

с помощью линейных непрерывных функционалов на $L_{p}(T)$ при условии, что функция $x(\cdot)$ известна в метрике $L_{p}(T)$ с точностью до $\gamma_{1}$. Это частный случай общей постановки задачи об оптимальном восстановлении линейных функционалов на классах по некоторой информации об элементах из этих классов, которая в нашем случае имеет вид:

$$
e\left(x^{(k)}(0), W_{p, r}^{n}\left(T, \gamma_{2}\right), \gamma_{1}\right)=\inf _{\varphi} \sup _{\substack{x(\cdot) \in W_{, r}^{n}\left(T, \gamma_{2}\right) \\\|x(\cdot)-y(\cdot)\|_{L_{p}(T)} \leqslant \gamma_{1}}}\left|x^{(k)}(0)-\varphi(y(\cdot))\right|,
$$

где нижняя грань берется по всем функциям (методам восстановления) $\varphi: L_{p}(T) \rightarrow \mathbb{R}$. Абстрактный вариант этой задачи в случае конечной информации и без погрешности впервые был рассмотрен Смоляком [18]. Он доказал, что если класс центрально симметричен, то среди оптимальных методов восстановления есть линейный. Этот вывод остается верен и в значительно более общей ситуации (см. [19]), когда, как в нашем случае, информация не является конечной и дается с погрешностью. Таким образом, если мы найдем решение задачи $\left(\mathrm{A}^{*}\right)$, то на самом деле мы решим задачу (В).

Если в задаче $\left(\mathrm{A}^{*}\right)$ погрешность $\gamma_{1}=0$, но при этом рассматриваются только функционалы $y^{*}$, норма которых не превосходит заданного числа $N$, то мы приходим к частной задаче Стечкина о приближении производных ограниченньми функционалами:

$$
E\left(N, x^{(k)}(0), W_{p, r}^{n}\left(T, \gamma_{2}\right)\right)=\inf _{\substack{y^{*} \in L_{p^{*}}(T) \\\left\|y^{*}\right\| \leqslant N}} \sup _{\substack{\|\|} \in W_{p, r}^{n}\left(T, \gamma_{2}\right)}\left|x^{(k)}(0)-\left\langle y^{*}, x(\cdot)\right\rangle\right| . \quad(\mathrm{C})
$$


Если обозначить через $S\left(\gamma_{1}\right)$ значение задачи $(\mathrm{A})$ как функцию $\gamma_{1}$, то всегда справедливо неравенство (см. [11])

$$
E\left(N, x^{(k)}(0), W_{p, r}^{n}\left(T, \gamma_{2}\right)\right) \geqslant \sup _{\gamma_{1} \geqslant 0}\left(S\left(\gamma_{1}\right)-N \gamma_{1}\right),
$$

которое также есть простое следствие двойственности выпуклых функций. Все три задачи (о неравенствах для производных, об оптимальном восстановлении и задача Стечкина) тесно связаны между собой, и это будет продемонстрировано далее на примерах. Но более подробно обсуждать тематику, связанную с оптимальным восстановлением функционалов и операторов, здесь не будем (предполагая в дальнейшем посвятить ей отдельную публикацию).

Общие принципы теории экстремальных задач, кратко изложенные выше, мы применяем к задачам типа (3) и надеемся убедить читателя в том, что за редчайшим исключением описанный стандартный подход не только естественным образом ведет к решению поставленной задачи, но и, как правило, позволяет увидеть возможные ее обобщения. Данная работа преследует еще одну цель: привлечь на примере решаемых здесь задач внимание коллег к началам обшей теории экстремума.

В первом параграфе работы доказываются неравенства для производных в классических постановках (и их естественные обобщения на $\mathbb{R}^{d}$ в случаях неравенств Харди-Литтлвуда-Полиа и Тайкова). Во втором параграфе рассматриваются разнообразные обобшения: задачи на других многообразиях, дробные производные и т.п. Кроме того, в $\S \S 1,2$ мы попутно решаем (как правило, в простейших ситуациях) связанные с неравенствами задачи оптимального восстановления функционалов и их наилучшего приближения ограниченньми (задача Стечкина).

\section{$\S 1$.}

Неравенство Харди-Литтлвуда-Полиа и его обобщения. В своей знаменитой монографии [4], изданной в 1934 году, Харди, Литтлвуд и Полиа показали, что $K_{\mathbb{R}}(n, k, 2,2,2)=1$. Перейдем к обобщению этого результата.

ЗАДАчА I (обобшенная задача Харди-Литтлвуда-Полиа - обшая задача о неравенствах для производных в $\left.L_{2}\left(\mathbb{R}^{d}\right)\right)$.

$$
\left\|D^{\alpha^{0}} x(\cdot)\right\|_{L_{2}\left(\mathbb{R}^{d}\right)} \rightarrow \max , \quad\left\|D^{\alpha^{j}} x(\cdot)\right\|_{L_{2}\left(\mathbb{R}^{d}\right)} \leqslant \gamma_{j}, \quad j=1, \ldots, N .
$$

Здесь $\alpha^{0}=\left(\alpha_{1}^{0}, \ldots, \alpha_{d}^{0}\right), \alpha_{j}=\left(\alpha_{1}^{j}, \ldots, \alpha_{d}^{j}\right) \in \mathbb{R}_{+}^{d}, \gamma_{j} \geqslant 0, j=1, \ldots, N, u$ $D^{\alpha} x(\cdot)$ для $\alpha=\left(\alpha_{1}, \ldots, \alpha_{d}\right) \in \mathbb{R}_{+}^{d}$ есть $\alpha$-я производная по Вейлю функции $x(\cdot) \in L_{2}\left(\mathbb{R}^{d}\right)$, определяемая по правилу: $D^{\alpha} x(\cdot)=\left(F^{-1} \circ \mathscr{E}^{\alpha} \circ F\right) x(\cdot)$, где $F u$ $F^{-1}$ - прямое и обратное преобразования Фурье в $L_{2}\left(\mathbb{R}^{d}\right)$, а $\mathscr{E} \alpha-$ оператор умножения на функцию $(i \tau)^{\alpha}=\left(i \tau_{1}\right)^{\alpha_{1}} \cdots\left(i \tau_{d}\right)^{\alpha_{d}},\left(i \tau_{j}\right)^{\alpha_{j}}=\left|\tau_{j}\right|^{\alpha_{j}} \exp \left\{\frac{1}{2} \pi i \operatorname{sgn} \tau_{j}\right\}$ $(\operatorname{sgn} 0=0), j=1, \ldots, d$. 
Решениє. 1) Формализация. Введем обозначение: $u(\cdot)=(2 \pi)^{-d}|F x(\cdot)|^{2}$ для $x(\cdot) \in L_{2}\left(\mathbb{R}^{d}\right)$. Тогда в силу равенства

$$
\|x(\cdot)\|_{L_{2}\left(\mathbb{R}^{d}\right)}^{2}=(2 \pi)^{-d}\|F x(\cdot)\|_{L_{2}\left(\mathbb{R}^{d}\right)}^{2}
$$

задача (i) (с заменой знака ее значения на противоположный) в образах Фурье запишется в виде

$$
\begin{gathered}
-\int_{\mathbb{R}^{d}}|\tau|^{2 \alpha^{0}} u(\tau) d \tau \rightarrow \min , \\
\int_{\mathbb{R}^{d}}|\tau|^{2 \alpha^{j}} u(\tau) d \tau \leqslant \gamma_{j}^{2}, \quad j=1, \ldots, N, \quad u(\tau) \geqslant 0 \text { п.в. }
\end{gathered}
$$

Напомним, что даже в простейшей ситуации (см. [4]), т.е. в самом неравенстве Харди-Литтлвуда-Полиа, решения не сушествует. Это побуждает расширить задачу, заменив функции мерами, т.е. положив $u(t) d t=d \mu(t)$. В этом случае задача (ii) перепишется следуюшим образом:

$$
\begin{gathered}
-\int_{\mathbb{R}^{d}}|t|^{2 \alpha^{0}} d \mu(t) \rightarrow \min \\
\int_{\mathbb{R}^{d}}|t|^{2 \alpha^{j}} d \mu(t) \leqslant \gamma_{j}^{2}, \quad j=1, \ldots, N, \quad d \mu(t) \geqslant 0 .
\end{gathered}
$$

2) Двойственность. Задача (ii') имеет вид задачи линейного программирования (LP) из введения. Выпишем (формально) двойственную к ней в соответствии $\mathrm{c}\left(\mathrm{LP}^{*}\right)\left(\lambda=\left(\lambda_{1}, \ldots, \lambda_{N}\right)\right)$ :

$$
\sum_{j=1}^{N} \lambda_{j} \gamma_{j}^{2} \rightarrow \max , \quad \lambda \leqslant 0, \quad|\tau|^{2 \alpha^{0}}+\sum_{j=1}^{N} \lambda_{j}|\tau|^{2 \alpha_{j}} \leqslant 0 \quad \forall \tau \in \mathbb{R}^{d} .
$$

Элементарно проверяется, что для любого допустимого вектора $\lambda$ в этой задаче и любой допустимой меры $d \mu(\cdot)$ в задаче $\left(\mathrm{ii}^{\prime}\right)$ справедливо неравенство

$$
\sum_{j=1}^{N} \lambda_{j} \gamma_{j}^{2} \leqslant-\int_{\mathbb{R}^{d}}|\tau|^{2 \alpha^{0}} d \mu(\tau)
$$

Если найдутся такие $\widehat{\lambda}$ и $d \widehat{\mu}(\cdot)$, на которых это неравенство обрашается в равенство, то понятно, что $d \widehat{\mu}(\cdot)$ будет решением задачи (ii'), а $\widehat{\lambda}$ - решением задачи (iii). Для нахождения таких $\hat{\lambda}$ и $d \widehat{\mu}(\cdot)$ рассмотрим следующую функцию на $\mathbb{R}^{d}$ :

$$
S(\alpha)=\min \left\{z \in \mathbb{R}:(\alpha, z) \in \operatorname{co}\left\{\left(\alpha^{1}, 2 \ln \gamma_{1}\right), \ldots,\left(\alpha^{N}, 2 \ln \gamma_{N}\right)\right\}\right\},
$$

где $\operatorname{co}\{A\}$ обозначает выпуклую оболочку множества $A$. Ясно, что функция $S(\cdot)$ вьпукла, конечна, если $\alpha^{0} \in \operatorname{co}\left\{\alpha^{1}, \ldots, \alpha^{N}\right\}$, и равна $+\infty$ в противном случае.

Пусть $\alpha^{0} \in \operatorname{co}\left\{\alpha^{1}, \ldots, \alpha^{N}\right\}$ и $z=\widehat{a}+\langle\alpha, \widehat{\xi}\rangle, \widehat{\xi}=\left(\widehat{\xi}_{1}, \ldots, \widehat{\xi}_{d}\right),-$ опорная гиперплоскость к графику функции $S(\cdot)$ в точке $\alpha^{0}$. Тогда, очевидно, найдутся такие точки $\left(\alpha^{j_{k}}, 2 \ln \gamma_{j_{k}}\right), k=1, \ldots, s$, из этой гиперплоскости, что $\alpha^{0}=\sum_{k=1}^{s} \theta_{j_{k}} \alpha^{j_{k}}$, 
где $\theta_{j_{k}}>0, k=1, \ldots, s$, и $\sum_{k=1}^{s} \theta_{j_{k}}=1$. Если теперь обозначить $\widehat{A}=\exp (\widehat{a})$ и выбрать $\widehat{\tau}=\left(\widehat{\tau}_{1}, \ldots, \widehat{\tau}_{d}\right) \in \mathbb{R}^{d}$ так, чтобы $\left|\widehat{\tau}_{i}\right|^{2}=\exp \left(\widehat{\xi}_{i}\right), i=1, \ldots, d$, то принадлежность опорной гиперплоскости указанных выше точек означает, что

$$
\widehat{A}|\widehat{\tau}|^{2 \alpha^{j_{k}}}=\gamma_{j_{k}}^{2}, \quad k=1, \ldots, s,
$$

т.е. мера $d \widehat{\mu}(\cdot)=\widehat{A} \delta(\cdot-\widehat{\tau})(\delta(\cdot-\widehat{\tau})-\delta$-функция в точке $\widehat{\tau})$ допустима в задаче $\left(\mathrm{ii}^{\prime}\right)$.

Положим далее $-\widehat{\lambda}_{j_{k}}=\theta_{j_{k}} \exp \left(-\left\langle\widehat{\xi}, \alpha^{j_{k}}-\alpha^{0}\right\rangle\right), k=1, \ldots, s$, и рассмотрим функцию на $\mathbb{R}^{d+1}$

$$
f(a, \xi)=-\exp \left(a+\left\langle\alpha^{0}, \xi\right\rangle\right)-\sum_{k=1}^{s} \widehat{\lambda}_{j_{k}} \exp \left(a+\left\langle\alpha^{j_{k}}, \xi\right\rangle\right) .
$$

Понятно, что $f(\cdot)$ - вьпуклая функция, и так как легко проверить, что $f(\widehat{a}, \widehat{\xi})=0$ и градиент $f(\cdot)$ в точке $(\widehat{a}, \widehat{\xi})$ равен нулю, то эта функция всюду неотрицательна на $\mathbb{R}^{d+1}$. Это означает, что функция $g(A, \tau)=-A|\tau|^{2 \alpha^{0}}-\sum_{k=1}^{s} \widehat{\lambda}_{j_{k}} A|\tau|^{2 \alpha^{j_{k}}}$ на $\mathbb{R}^{d+1}$ также всюду неотрицательна и

$$
g(\widehat{A}, \widehat{\tau})=0 .
$$

Если теперь определить вектор $\hat{\lambda} \in \mathbb{R}^{N}$ с компонентами $\widehat{\lambda}_{j_{k}}$, полагая остальные компоненты нулями, то (b) (с учетом (a)) означает, что на $\widehat{\lambda}$ и $d \widehat{\mu}(\cdot)$ неравенство (iv) обрашается в равенство. Итак, $d \widehat{\mu}(\cdot)$ - решение задачи (ii'). Ее значение в силу определений равно

$$
\begin{aligned}
-\int_{\mathbb{R}^{d}}|t|^{2 \alpha^{0}} d \widehat{\mu}(t)=-\widehat{A}|\widehat{\tau}|^{2 \alpha^{0}}=-\exp \left(\widehat{a}+\left\langle\alpha^{0}, \widehat{\xi}\right\rangle\right)=-\exp S\left(\alpha^{0}\right) \\
\quad=-\min \left\{\prod_{j=1}^{N} \gamma_{j}^{2 \theta_{j}}: \theta_{j} \geqslant 0, j=1, \ldots, N, \sum_{j=1}^{N} \theta_{j}=1, \alpha^{0}=\sum_{j=1}^{N} \theta_{j} \alpha^{j}\right\} .
\end{aligned}
$$

Эта величина, очевидно, дает оценку снизу для значения задачи (ii). Но эта оценка точна, так как мы можем выбрать последовательность функций $\left\{x_{n}(\cdot)\right\}$, $x_{n}(\cdot) \in L_{2}\left(\mathbb{R}^{d}\right)$, такую, что $(2 \pi)^{d}\left|F x_{n}(\cdot)\right|^{2} \rightarrow \widehat{A} \delta(t-\widehat{\tau})$ при $n \rightarrow \infty$. Таким образом, задача (ii) решена, если $\alpha^{0} \in \operatorname{co}\left\{\alpha^{1}, \ldots, \alpha^{N}\right\}$.

Пусть теперь $\alpha^{0} \notin \operatorname{co}\left\{\alpha^{1}, \ldots, \alpha^{N}\right\}$. Тогда $\forall M>0$ точка $\left(\alpha^{0}, M\right) \notin$ ері $S$. Следовательно, существует гиперплоскость $z=\bar{a}+\langle\alpha, \bar{\xi}\rangle$, которая разделяет множество ері $S$ и точку $\left(\alpha^{0}, M\right)$. Очевидно, что

$$
\begin{gathered}
\bar{a}+\left\langle\alpha^{j}, \bar{\xi}\right\rangle \leqslant 2 \ln \gamma_{j}, \quad j=1, \ldots, N, \\
\bar{a}+\left\langle\alpha^{0}, \bar{\xi}\right\rangle \geqslant M .
\end{gathered}
$$

Если теперь обозначить $\bar{A}=\exp (\bar{a})$ и взять такую точку $\bar{\tau}=\left(\bar{\tau}_{1}, \ldots, \bar{\tau}_{d}\right) \in \mathbb{R}^{d}$, что $\left|\bar{\tau}_{i}\right|^{2}=\exp \left(\bar{\xi}_{i}\right), i=1, \ldots, d$, то соотношения (c) и (d) перепишутся, соответственно, в виде:

$$
\begin{gathered}
\bar{A}|\bar{\tau}|^{2 \alpha^{j}} \leqslant \gamma_{j}^{2}, \quad j=1, \ldots, N, \\
\bar{A}|\bar{\tau}|^{2 \alpha^{0}} \geqslant \exp (M) .
\end{gathered}
$$

Это означает, что мера $d \bar{\mu}(\cdot)=\bar{A} \delta(\cdot-\bar{\tau})$ допустима в задаче $\left(\mathrm{ii}^{\prime}\right)$, и в силу произвольности $M>0$ значение задачи равно $-\infty$. Понятно, что тогда и значение задачи (ii) равно $-\infty$.

Итак, доказана следуюшая 
Teоpema I. Пусть в задаче I $\alpha^{0}, \alpha^{j} \in \mathbb{R}_{+}^{d}, \gamma_{j}>0, j=1, \ldots, N$. Обозначим через $S_{\mathrm{I}}=S_{\mathrm{I}}\left(\alpha^{0}, \alpha^{1}, \ldots, \alpha^{N}, \gamma_{1}, \ldots, \gamma_{N}\right)$ ее значение. Тогда

1) если $\alpha^{0} \in \operatorname{co}\left\{\alpha^{1}, \ldots, \alpha^{N}\right\}$, mo

$$
S_{\mathrm{I}}=\min \left\{\prod_{j=1}^{N} \gamma_{j}^{\theta_{j}}: \theta_{j} \geqslant 0, j=1, \ldots, N, \sum_{j=1}^{N} \theta_{j}=1, \alpha^{0}=\sum_{j=1}^{N} \theta_{j} \alpha^{j}\right\} ;
$$

2) если $\alpha^{0} \notin \operatorname{co}\left\{\alpha^{1}, \ldots, \alpha^{N}\right\}$, mo $S_{\mathrm{I}}=+\infty$.

3) Решение в задаче I не существует. Существует лищь обобщенное решение, являющееся преобразованием Фурье меры $d \widehat{\mu}(\cdot)=\widehat{A} \delta(\cdot-\widehat{\tau})$, описанной выше.

ЗАмечАнИЕ. Отметим, что проведенные выше рассуждения решают фактически и более общую задачу, чем (i):

$$
\left\|D^{\alpha^{0}} x(\cdot)\right\|_{L_{2}\left(\mathbb{R}^{d}\right)} \rightarrow \max , \quad\left\|D^{\alpha^{j}} x(\cdot)\right\|_{L_{2}\left(\mathbb{R}^{d}\right)} \leqslant \gamma_{j}, \quad \alpha^{j} \in K,
$$

где $K$ - компакт в $\mathbb{R}_{+}^{d}$. Ответ по смыслу тот же: если $\alpha^{0} \in \operatorname{co} K$, то значение задачи $\left(\mathrm{i}^{\prime}\right)$ конечно и в соответствуюшем выражении берется минимум по всем симплексам из $K$, которые содержат $\alpha^{0}$; если же $\alpha^{0} \notin$ со $K$, то значение этой задачи равно $+\infty$.

II. Неравенство Тайкова и его обобщения. В 1968 году Тайковым [6] для любых $k \in \mathbb{Z}_{+}, n \in \mathbb{N}, k<n$, и при $p=r=2, q=\infty, T=\mathbb{R}$ было доказано точное неравенство (1) с константой

$$
K_{\mathbb{R}}(n, k, 2, \infty, 2)=\left(2 n \sin \frac{2 k+1}{2 n} \pi\right)^{-\frac{1}{2}}\left(\frac{2 n}{2(n-k)-1}\right)^{\frac{2(n-k)-1}{4 n}}\left(\frac{2 n}{2 k+1}\right)^{\frac{2 k+1}{4 n}} .
$$

Рассмотрим следующее обобщение этого результата.

ЗАДАчА II (обобщенная задача Тайкова - общая задача о неравенствах для производных в $C^{b}\left(\mathbb{R}^{d}\right)$ при ограничениях в $\left.L_{2}\left(\mathbb{R}^{d}\right)\right)$.

$$
\left\|D^{\alpha^{0}} x(\cdot)\right\|_{C^{b}\left(\mathbb{R}^{d}\right)} \rightarrow \max , \quad\left\|D^{\alpha^{j}} x(\cdot)\right\|_{L_{2}\left(\mathbb{R}^{d}\right)} \leqslant \gamma_{j}, \quad j=1, \ldots, N .
$$

Здесь $\alpha^{0}, \alpha^{j}, \gamma_{j}, j=1, \ldots, N$, и операторь дробного дифференцирования имеют тот же смысл, что и в задаче I.

Решение. 1) Формализация. Ясно, что значение задачи (i) не уменшшися, если максимизировать не норму функции $D^{\alpha^{0}} x(\cdot)$, а модуль ее значения в нуле. Тогда, переходя к образам Фурье и используя равенство Парсеваля, эта задача перепишется в виде

$$
\begin{gathered}
\left|\int_{\mathbb{R}^{d}}(i \tau)^{\alpha^{0}} F x(\tau) d \tau\right| \rightarrow \max , \\
\int_{\mathbb{R}^{d}}|\tau|^{2 \alpha^{j}}|F x(\tau)|^{2} d \tau \leqslant(2 \pi)^{d} \gamma_{j}^{2}, \quad j=1, \ldots, N .
\end{gathered}
$$


Обозначая $w(\tau)=\exp (i \xi(\tau)) F x(\tau)=u(\tau)+i v(\tau)$, нетрудно проверить, что задача (ii) (с точностью до замены знака ее значения на противоположный) равносильна следующей задаче:

$$
\begin{gathered}
-\int_{\mathbb{R}^{d}}|\tau|^{\alpha^{0}} u(\tau) d \tau \rightarrow \min , \\
\int_{\mathbb{R}^{d}}|\tau|^{2 \alpha^{j}} u^{2}(\tau) d \tau \leqslant(2 \pi)^{d} \gamma_{j}^{2}, \quad j=1, \ldots, N .
\end{gathered}
$$

Это задача выпуклого (и даже квадратичного) программирования.

2) Двойственность. Снова воспользуемся двойственностью, применяя непосредственно теорему Фенхеля-Моро. Для этого заметим сначала, что если $\alpha^{0} \in \operatorname{int} P$, где $P=\operatorname{co}\left\{\alpha^{1}, \ldots, \alpha^{N}\right\}-\left(\frac{1}{2}, \ldots, \frac{1}{2}\right)$, то несложный анализ показывает, что интеграл

$$
J=\int_{\mathbb{R}^{d}}|t|^{2 \alpha^{0}}\left(\sum_{j=1}^{N}|t|^{2 \alpha^{j}}\right)^{-1} d t
$$

конечен (см. [20]). Отсюда, используя неравенство Коши-Буняковского и равенство Парсеваля, получаем, что

$$
\left\|D^{\alpha^{0}} x(\cdot)\right\|_{C^{b}\left(\mathbb{R}^{d}\right)} \leqslant J^{1 / 2}\left(\sum_{j=1}^{N}\left\|D^{\alpha^{j}} x(\cdot)\right\|_{L_{2}\left(\mathbb{R}^{d}\right)}^{2}\right)^{1 / 2} .
$$

Таким образом, если $\alpha^{0} \in \operatorname{int} P$, то значение задачи (i) конечно для любых $\gamma_{j} \geqslant 0$, $j=1, \ldots, N$.

Пусть $\delta=\left(\delta_{1}, \ldots, \delta_{N}\right) \in \mathbb{R}^{N}$. Рассмотрим задачу (ii'), где вместо $(2 \pi)^{d} \gamma_{j}^{2}$ стоит $\delta_{j}, j=1, \ldots, N$, и обозначим через $S(\delta)$ ее значение. Ясно, что $S(\cdot)-$ вьпуклая функция на $\mathbb{R}^{N}$ со значениями в $\mathbb{R} \cup\{+\infty\}$ и нетрудно видеть, что она замкнута. Поэтому по теореме Фенхеля-Моро $S(\cdot)=S^{* *}(\cdot)$. Найдем сначала первую сопряженную к $S(\cdot)$, обозначая для краткости

$$
f_{0}(u(\cdot))=\int_{\mathbb{R}^{d}}|t|^{\alpha^{0}} u(t) d t, \quad f_{j}(u(\cdot))=\int_{\mathbb{R}^{d}}|t|^{2 \alpha^{j}} u(t) d t, \quad j=1, \ldots, N .
$$

По определению $\left(\beta=\left(\beta_{1}, \ldots, \beta_{N}\right)\right)$

$$
\begin{aligned}
S^{*}(\beta) & =\sup _{\delta \in \mathbb{R}^{N}}(\langle\beta, \delta\rangle-S(\delta))=\sup _{\delta \in \mathbb{R}^{N}} \sup _{\left\{u(\cdot): f_{j}(u(\cdot)) \leqslant \delta_{j}\right\}}\left(\langle\beta, \delta\rangle+f_{0}(u(\cdot))\right) \\
& =\sup _{\left\{u(\cdot): f_{j}(u(\cdot))<\infty\right\}\left\{\delta \in \mathbb{R}^{N}: \delta_{j} \geqslant f_{j}(u(\cdot))\right\}}\left(\langle\beta, \delta\rangle+f_{0}(u(\cdot))\right) \\
& =\sup _{\left\{u(\cdot): f_{j}(u(\cdot))<\infty\right\}} \int_{\mathbb{R}^{d}}\left(\sum_{j=1}^{N} \beta_{j}|t|^{2 \alpha^{j}} u^{2}(t)+|t|^{\alpha^{0}} u(t)\right) d t,
\end{aligned}
$$

если $\beta_{j} \leqslant 0, j=1, \ldots, N$, и $S^{*}(\beta)=+\infty$ в противном случае. 
Понятно, что верхняя грань в последнем интеграле достигается на функции

$$
u(t)=-|t|^{\alpha^{0}}\left(2 \sum_{j=1}^{N} \beta_{j}|t|^{2 \alpha^{j}}\right)^{-1}
$$

и, следовательно, значение этой грани равно

$$
-\frac{1}{4} \int_{\mathbb{R}^{d}}|t|^{2 \alpha^{0}}\left(\sum_{j=1}^{N} \beta_{j}|t|^{2 \alpha^{j}}\right)^{-1} d t
$$

Таким образом, если $S_{\mathrm{II}}=S_{\mathrm{II}}\left(\alpha^{0}, \alpha^{1}, \ldots, \alpha^{N}, \gamma_{1}, \ldots, \gamma_{N}\right)$ - значение задачи II и $\alpha^{0} \in \operatorname{int} P$, то по теореме Фенхеля-Моро

$$
S_{\mathrm{II}}=\inf _{\substack{\lambda_{j} \geqslant 0 \\ j=1, \ldots, N}}\left((2 \pi)^{d} \sum_{j=1}^{N} \lambda_{j} \gamma_{j}^{2}+\frac{1}{4} \int_{\mathbb{R}^{d}} \frac{|t|^{2 \alpha^{0}}}{\sum_{j=1}^{N} \lambda_{j}|t|^{2 \alpha^{j}}} d t\right)
$$

Это конечномерная задача и для любых значений параметров имеет смысл вопрос о нахождении ее приближенного решения.

Отметим теперь, что если $\alpha^{0} \notin \operatorname{int} P$, то снова несложные оценки (см. [20]) показывают, что для любых $\beta_{j}, j=1, \ldots, N$, интеграл

$$
\int_{\mathbb{R}^{d}}|t|^{2 \alpha^{0}}\left(\sum_{j=1}^{N} \beta_{j}|t|^{2 \alpha^{j}}\right)^{-1} d t
$$

равен бесконечности. Тогда нетрудно проверить, что в этом случае $S^{* *}(\delta) \equiv+\infty$, и так как всегда $S(\cdot) \geqslant S^{* *}(\cdot)$, то $S(\delta) \equiv+\infty$.

Когда $N=d+1$, векторы $\alpha^{1}, \ldots, \alpha^{d+1}$ аффинно независимы и $\alpha^{0} \in \operatorname{int} P$, то нетрудно выписать явное выражение для значения задачи.

Итак, справедлива следующая

Teоpema II. Пусть в задаче II $\alpha^{0}, \alpha^{j} \in \mathbb{R}_{+}^{d}, \gamma_{j}>0, j=1, \ldots, N$. Тогда

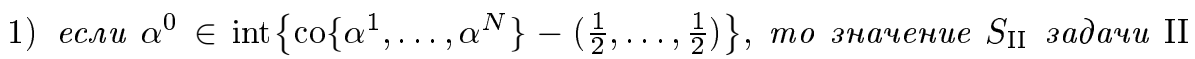
дается формулой (iii);

2) если $\alpha^{0} \notin \operatorname{int}\left\{\operatorname{co}\left\{\alpha^{1}, \ldots, \alpha^{N}\right\}-\left(\frac{1}{2}, \ldots, \frac{1}{2}\right)\right\}$, mo $S_{\text {II }}=+\infty$;

3) если $N=d+1$, векторы $\alpha^{1}, \ldots, \alpha^{d+1}$ аффинно независимы и вектор $\alpha^{0}=\sum_{j=1}^{d+1} \theta_{j}\left(\alpha^{j}-\left(\frac{1}{2}, \ldots, \frac{1}{2}\right)\right)$, əде $\theta_{j}>0, j=1, \ldots, d+1, \sum_{j=1}^{d+1} \theta_{j}=1$, mo

$$
S_{\mathrm{II}}=\frac{1}{2 \pi}\left(\int_{\mathbb{R}^{d}} \frac{|t|^{2 \alpha^{0}}}{\sum_{j=1}^{N} \theta_{j}|t|^{2 \alpha^{j}}} d t\right)^{1 / 2} .
$$

По поводу этого результата см. также [20]. 
III. Неравенство Габушина. В 1969 году Габушиным [9] для любых $k \in \mathbb{Z}_{+}$, $n \in \mathbb{N}, k<n$, при $p=r=2, q=\infty, T=\mathbb{R}_{+}$было доказано точное неравенство (1). Наилучшая константа выражается через решение некоторой системы линейных уравнений.

Докажем этот результат сначала для простейшего случая $n=1$ и для этого же случая решим (попутно) задачи, связанные с оптимальным восстановлением функционала $x(\cdot) \mapsto x(0)$ и его наилучшим приближением ограниченными функционалами (задача Стечкина). Итак, мы рассматриваем следуюшую задачу на $\mathscr{W}_{2}^{1}\left(\mathbb{R}_{+}\right)$:

$$
\|x(\cdot)\|_{C^{b}\left(\mathbb{R}_{+}\right)} \rightarrow \max , \quad\|x(\cdot)\|_{L_{2}\left(\mathbb{R}_{+}\right)} \leqslant \delta, \quad\|\dot{x}(\cdot)\|_{L_{2}\left(\mathbb{R}_{+}\right)} \leqslant 1 .
$$

Константы $\gamma_{1}=\delta$ и $\gamma_{2}=1$ (см. (2)) выбраны для более наглядной связи с задачей восстановления (см. ниже).

РЕшениЕ. 1) Формализация. В качестве таковой рассматриваем задачу

$$
-x(0) \rightarrow \min , \quad \int_{\mathbb{R}_{+}} x^{2}(t) d t \leqslant \delta^{2}, \quad \int_{\mathbb{R}_{+}} \dot{x}^{2}(t) d t \leqslant 1,
$$

решения которой, очевидно, являются и решениями (i), а значения этих задач отличаются знаком.

2) Принцип Лагранжа. Задача (ii) - это задача вариационного исчисления и одновременно выпуклого программирования. Ее функция Лагранжа имеет вид:

$$
\mathscr{L}=\lambda_{0} x(0)+\lambda_{1}\left(\int_{\mathbb{R}_{+}} x^{2}(t) d t-\delta^{2}\right)+\lambda_{2}\left(\int_{\mathbb{R}_{+}} \dot{x}^{2}(t) d t-1\right) .
$$

Можно считать, что $\lambda_{0}=1$. Если $\widehat{x}(\cdot)$ - решение задачи, то найдутся такие $\lambda_{1} \geqslant 0$ и $\lambda_{2} \geqslant 0$, что $\widehat{x}(\cdot)$ удовлетворяет уравнению Эйлера

$$
\lambda_{2} \ddot{x}-\lambda_{1} x=0,
$$

условиям трансверсальности

$$
2 \lambda_{2} \dot{x}(0)=-1, \quad \lim _{t \rightarrow \infty} \lambda_{2} \dot{x}(t)=0
$$

и условиям дополняющей нежесткости

$$
\lambda_{1}\left(\int_{\mathbb{R}_{+}} x^{2}(t) d t-\delta^{2}\right)=0, \quad \lambda_{2}\left(\int_{\mathbb{R}_{+}} \dot{x}^{2}(t) d t-1\right)=0 .
$$

3) Исследование. Из (а) и (b) следует, что $\lambda_{i}>0, i=1,2$. Обшее решение (a) есть

$$
x(t)=c_{1} \exp \left(\sqrt{\lambda_{1} / \lambda_{2}} t\right)+c_{2} \exp \left(-\sqrt{\lambda_{1} / \lambda_{2}} t\right) .
$$

Из (b) вытекает, что $c_{1}=0$ и $c_{2}=1 /\left(2 \sqrt{\lambda_{1} \lambda_{2}}\right)$. Поскольку $\lambda_{i}>0, i=1,2$, то из (c) следует, что на экстремали ограничения в (ii) обрашаются в равенства, откуда 
получаем, что $\lambda_{1}=1 /(2 \delta \sqrt{2 \delta})$ и $\lambda_{2}=\sqrt{\delta} /(2 \sqrt{2})$. Итак, если $\widehat{x}(\cdot)-$ решение задачи (ii), то оно имеет вид:

$$
\widehat{x}(t)=\sqrt{2 \delta} e^{-t / \delta} .
$$

Функция Лагранжа как функция $x(\cdot)$ для найденных $\lambda_{1}$ и $\lambda_{2}$ достигает своего минимума в точке $\widehat{x}(\cdot)$, и поэтому ее производная в данной точке равна нулю. Это равносильно тому, что для всех $x(\cdot) \in \mathscr{W}_{2}^{1}\left(\mathbb{R}_{+}\right)$справедливо равенство

$$
x(0)=\frac{1}{\delta} \int_{\mathbb{R}_{+}} e^{-t / \delta} x(t) d t-\int_{\mathbb{R}_{+}} e^{-t / \delta} \dot{x}(t) d t
$$

(которое, впрочем, тривиально проверяется и непосредственно). Теперь, используя только это тождество, мы решим три задачи:

А) о точном мультипликативном неравенстве;

В) об оптимальном методе восстановления функционала $x(\cdot) \mapsto x(0)$;

C) о наилучшем приближении этого функционала ограниченными (задача Стечкина).

А) Оценивая по неравенству Коши-Буняковского интегралы справа в (iii), получаем, что для всех $x(\cdot) \in \mathscr{W}_{2}^{1}\left(\mathbb{R}_{+}\right)$, допустимых в (ii), справедливо неравенство $|x(0)| \leqslant \sqrt{2 \delta}$, которое является точньм, так как на функции $\widehat{x}(\cdot)$ оно обрашается в равенство. Отсюда следует, что $\widehat{x}(\cdot)$ - решение задачи (ii) и, тем самым, задачи (i). Отсюда же и из элементарных соображений однородности вытекает, что для всех $x(\cdot) \in \mathscr{W}_{2}^{1}\left(\mathbb{R}_{+}\right)$выполняется точное неравенство

$$
\|x(\cdot)\|_{C^{b}\left(\mathbb{R}_{+}\right)} \leqslant \sqrt{2}\|x(\cdot)\|_{L_{2}\left(\mathbb{R}_{+}\right)}^{1 / 2}\|\dot{x}(\cdot)\|_{L_{2}\left(\mathbb{R}_{+}\right)}^{1 / 2}
$$

т.е. $K_{\mathbb{R}_{+}}(1,0,2, \infty, 2)=\sqrt{2}$.

В) Двойственная задача к (ii) (см. задачи (А) и (A*)) есть задача оптимального восстановления функционала $x(\cdot) \mapsto x(0)$ на классе

$$
W_{2}^{1}\left(\mathbb{R}_{+}\right)=\left\{x(\cdot) \in \mathscr{W}_{2}^{1}\left(\mathbb{R}_{+}\right):\|\dot{x}(\cdot)\|_{L_{2}\left(\mathbb{R}_{+}\right)} \leqslant 1\right\}
$$

при условии, что функция $x(\cdot)$ в метрике $L_{2}\left(\mathbb{R}_{+}\right)$известна с точностью до $\delta$. Функционал $y^{*}$ на $L_{2}\left(\mathbb{R}_{+}\right)$, который сопоставляет $y(\cdot) \in L_{2}\left(\mathbb{R}_{+}\right)$первое слагаемое в (iii) (с заменой $x(\cdot)$ на $y(\cdot))$, и есть оптимальный метод восстановления. Действительно, обозначая $\xi(\cdot)=x(\cdot)-y(\cdot)$, используя (iii) и снова неравенство Коши-Буняковского, получаем

$$
\begin{aligned}
x(0)-\left\langle y^{*}, y(\cdot)\right\rangle & =x(0)-\frac{1}{\delta} \int_{\mathbb{R}_{+}} e^{-t / \delta} y(t) d t \\
& =x(0)-\frac{1}{\delta} \int_{\mathbb{R}_{+}} e^{-t / \delta} x(t) d t+\frac{1}{\delta} \int_{\mathbb{R}_{+}} e^{-t / \delta} \xi(t) d t \\
& =-\int_{\mathbb{R}_{+}} e^{-t / \delta} \dot{x}(t) d t+\frac{1}{\delta} \int_{\mathbb{R}_{+}} e^{-t / \delta} \xi(t) d t \leqslant \sqrt{2 \delta} .
\end{aligned}
$$


Величина справа есть $|\widehat{x}(0)|$, и так как значение двойственной задачи всегда не превосходит значение исходной, то $y^{*}$ - оптимальный метод восстановления функционала $x(\cdot) \mapsto x(0)$ на классе $W_{2}^{1}\left(\mathbb{R}_{+}\right)$. Этот факт мы записьваем следующим образом:

$$
x(0) \approx \frac{1}{\delta} \int_{\mathbb{R}_{+}} e^{-t / \delta} y(t) d t .
$$

Кроме того (см. (В)),

$$
e\left(x(0), W_{2}^{1}\left(\mathbb{R}_{+}\right), \delta\right)=\sqrt{2 \delta} .
$$

С) Пусть $N>0$. Функционал $x^{*}$ на $L_{2}\left(\mathbb{R}_{+}\right)$, который сопоставляет функции $x(\cdot) \in L_{2}\left(\mathbb{R}_{+}\right)$первое слагаемое в (iii) с $\delta=1 /\left(2 N^{2}\right)$, имеет, очевидно, норму $N$, и мы получаем (используя (iii))

$$
\left|x(0)-\left\langle x^{*}, x(\cdot)\right\rangle\right|=\left|x(0)-2 N^{2} \int_{\mathbb{R}_{+}} e^{-2 N^{2} t} d t\right|=\left|\int_{\mathbb{R}_{+}} e^{-2 N^{2} t} \dot{x}(t) d t\right| \leqslant \frac{1}{2 N},
$$

т.е. $E\left(N, x(0), W_{2}^{1}\left(\mathbb{R}_{+}\right)\right) \leqslant 1 /(2 N)$. С другой стороны, величина справа в $(\mathrm{D})$, как легко подсчитать, также равна $1 /(2 N)$ (в нашем случае $\left.S\left(\gamma_{1}\right)=\sqrt{2 \gamma_{1}}\right)$. Следовательно, $E\left(N, x(0), W_{2}^{1}\left(\mathbb{R}_{+}\right)\right)=1 /(2 N)$ и, значит, $x^{*}$ - наилучшее приближение для неограниченного функционала $x(\cdot) \mapsto x(0)$ на классе $W_{2}^{1}\left(\mathbb{R}_{+}\right)$.

В обшем случае (т.е. для любых $n \in \mathbb{N}$ и $0 \leqslant k<n$ ) те же рассуждения приводят к решению соответствуюших задач (разумеется, не в столь явном виде). Однако, ограничимся здесь только задачей о неравенствах для производных и ради простоты вычислений несколько иначе формализуем исходную задачу, чтобы иметь дело лишь с одним множителем Лагранжа.

РЕшениЕ. 1) Формализация. Рассмотрим следуюшую экстремальную задачу на $\mathscr{W}_{2}^{n}\left(\mathbb{R}_{+}\right)$:

$$
\int_{\mathbb{R}_{+}}\left(x^{2}(t)+\left(x^{(n)}(t)\right)^{2}\right) d t \rightarrow \min , \quad x^{(k)}(0)=1 .
$$

2) Принцип Лагранжа. Задача (i) относится к вариационному исчислению и одновременно к выпуклому программированию. Ее функция Лагранжа такова:

$$
\begin{gathered}
\mathscr{L}=\int_{\mathbb{R}_{+}}\left(\left(x_{1}^{2}+u^{2}\right)+p_{1}\left(\dot{x}_{1}-x_{2}\right)+\cdots+p_{n}\left(\dot{x}_{n}-u\right)\right) d t+\lambda x_{k+1}(0), \\
x=x_{1}, \quad \dot{x}_{1}=x_{2}, \quad \ldots, \quad \dot{x}_{n-1}=x_{n}, \quad \dot{x}_{n}=u
\end{gathered}
$$

Если $\widehat{x}(\cdot)$ - решение (i), то найдутся такие вектор-функция $p$ и число $\lambda$, что $\widehat{x}(\cdot)$ удовлетворяет уравнениям $\dot{p}_{1}=x_{1}, \dot{p}_{2}=-p_{1}, \ldots, \dot{p}_{n}=-p_{n-1}, p_{n}=u$, которые сводятся к уравнению Эйлера-Пуассона

$$
(-1)^{n} x^{(2 n)}+x=0
$$

и условиям трансверсальности

$$
\begin{gathered}
p_{i}(0)=0, \quad i=1, \ldots, k, k+2, \ldots, n, \quad p_{k+1}=\lambda, \\
\lim _{t \rightarrow \infty} p_{i}(t)=0, \quad i=1, \ldots, n .
\end{gathered}
$$


3) Исследование. Характеристический многочлен уравнения (a), очевидно, не содержит чисто мнимых корней. В силу (b) в представлении $\widehat{x}(\cdot)$ отсутствуют слагаемые, соответствующие корням характеристического многочлена с положительными действительными частями. Если $\zeta_{1}, \ldots, \zeta_{n}-$ корни этого многочлена с отрицательными действительньми частями, то ясно, что $\widehat{x}(\cdot)$ должно удовлетворять уравнению

$$
x^{(n)}+\sum_{j=1}^{n} \nu_{j} x^{(n-j)}=0
$$

где числа $\nu_{1}, \ldots, \nu_{n}$ однозначно определяются данными корнями. Дифференцируя последовательно уравнение (a) $(n-1)$ раз и используя условия (b), получим, что числа $\widehat{x}(0), \dot{\widehat{x}}(0), \ldots, \widehat{x}^{(k-1)}(0), \widehat{x}^{(k+1)}(0), \ldots, \widehat{x}^{(n-1)}(0)\left(\widehat{x}^{(k)}(0)=1\right.$ по условию) и $\lambda$ должны удовлетворять следуюшей системе линейных уравнений:

$$
\sum_{j=s}^{n} \nu_{j} x^{(n-j+s-1)}(0)= \begin{cases}0, & 1 \leqslant s \leqslant n, s \neq n-k \\ (-1)^{n-k} \lambda, & s=n-k\end{cases}
$$

с треугольной матрищей.

Решая уравнение (c) операторным методом, получаем, что преобразование Лапласа его решения имеет вид $X(p)=P(p) / Q(p)$, где $P(\cdot)$ - полином степени $(n-1)$, коэффициенты которого однозначно определяются через начальные значения функции $\widehat{x}(\cdot)$, найденные из $(\mathrm{d})$, а $Q(p)=p^{n}+\sum_{j=1}^{n} \mu_{j} p^{n-j}$. Используя формулу обращения, приходим к следуюшему результату

Tеорема III. Решением задачи (i) является функиия

$$
\widehat{x}(t)=\sum_{j=1}^{n} \frac{P\left(\zeta_{j}\right)}{Q^{\prime}\left(\zeta_{j}\right)} e^{\zeta_{j} t} .
$$

Доказательство легко следует из условий достаточности в задачах выпуклого программирования. Переход от задачи (i) к соответствуюшему точному мультипликативному неравенству осушествляется стандартным пересчетом.

IV. Неравенство Любича-Купцова. В 1960 году Любич опубликовал статью [7], в которой (среди прочего) содержалось решение задачи о неравенствах для производных на полупрямой для $p=q=r=2$. Эта работа осталась незамеченной многими специалистами по неравенствам, и этим, по-видимому, объясняется то, что Купов, опубликовавший в 1975 году решение той же задачи [8], считал, что делает это впервые.

Обсуждение этой темы мы начнем с простейшего примера (изложенного еще в монографии Харди, Литтлвуд, Полиа [4]), когда $n=2, k=1$ :

$$
\|\dot{x}(\cdot)\|_{L_{2}\left(\mathbb{R}_{+}\right)} \rightarrow \max , \quad\|x(\cdot)\|_{L_{2}\left(\mathbb{R}_{+}\right)} \leqslant 1, \quad\|\ddot{x}(\cdot)\|_{L_{2}\left(\mathbb{R}_{+}\right)} \leqslant 1 .
$$

Решим его “стандартно", и это решение даст нам ключ к получению и результата Любича-Купюва. 
РЕшениЕ. 1) Формализация. Снова сводим нашу задачу к задаче классического вариационного исчисления

$$
\int_{\mathbb{R}_{+}} \dot{x}^{2}(t) d t \rightarrow \max , \quad \int_{\mathbb{R}_{+}}\left(x^{2}(t)+\ddot{x}^{2}(t)\right) d t \leqslant 1
$$

2) Принцип Лагранжа. Применение этого принципа приводит к следующим соотношениям. Сушествует число $\mu>0$ такое, что удовлетворяются уравнение Эйлера-Пуассона

$$
x^{(4)}+\mu \ddot{x}+x=0
$$

и условия трансверсальности

$$
x^{(3)}(0)+\mu \dot{x}(0)=0, \quad \ddot{x}(0)=0 .
$$

3) Исследование. Характеристический многочлен $z^{4}+\mu z^{2}+1$ разлагается на два сомножителя, один из которых $z^{2}+\nu z+1(\nu=\sqrt{2-\mu})$ имеет корни в левой полуплоскости. Значит, решение задачи удовлетворяет дифференциальному уравнению

$$
\ddot{x}+\nu \dot{x}+x=0 .
$$

Дифференцируя (c) и подставляя $t=0$, получаем (учитывая (b)) равенство $x^{(3)}(0)+\dot{x}(0)=0$, откуда и из (b) извлекаем, что $\mu=1$, а тем самьм, и $\nu=1$. Решая теперь уравнение $\ddot{x}+\dot{x}+x=0$ с краевьм условием $\dot{x}(0)+x(0)=0$, получаем семейство решений

$$
x(t)=A e^{-t / 2} \cos \left(\frac{\sqrt{3}}{2} t+\frac{\pi}{6}\right),
$$

где константа $A$ находится из изопериметрического условия.

Легко проверить, что для всех $x(\cdot) \in \mathscr{W}_{2}^{2}\left(\mathbb{R}_{+}\right)$справедливо равенство

$$
\int_{\mathbb{R}_{+}}\left(\ddot{x}^{2}(t)-\dot{x}^{2}(t)+x^{2}(t)\right) d t=\int_{\mathbb{R}_{+}}(\ddot{x}(t)+\dot{x}(t)+x(t))^{2} d t+(x(0)+\dot{x}(0))^{2}
$$

(на самом деле, это формула Вейеритрасса в вариационном исчислении, см., например, [12]), из которого вытекает неравенство

$$
\int_{\mathbb{R}_{+}} \dot{x}^{2}(t) d t \leqslant \int_{\mathbb{R}_{+}}\left(x(t)+\ddot{x}^{2}(t)\right) d t
$$

обращающееся в равенство на функциях (d). Из этого неравенства, в свою очередь, следует точное мультипликативное неравенство

$$
\|\dot{x}(\cdot)\|_{L_{2}\left(\mathbb{R}_{+}\right)} \leqslant 2\|x(\cdot)\|_{L_{2}\left(\mathbb{R}_{+}\right)}^{1 / 2}\|\ddot{x}(\cdot)\|_{L_{2}\left(\mathbb{R}_{+}\right)}^{1 / 2} \quad \forall x(\cdot) \in \mathscr{W}_{2}^{2}\left(\mathbb{R}_{+}\right) .
$$

Переходим собственно к задаче Любича-Купцова:

$$
\left\|x^{(k)}(\cdot)\right\|_{L_{2}\left(\mathbb{R}_{+}\right)} \rightarrow \max , \quad\|x(\cdot)\|_{L_{2}\left(\mathbb{R}_{+}\right)} \leqslant 1, \quad\left\|x^{(n)}(\cdot)\right\|_{L_{2}\left(\mathbb{R}_{+}\right)} \leqslant 1 .
$$


РЕшениє. 1) Формализация. Как и в простейшем случае, редуцируем задачу (i) к следуюшей задаче вариационного исчисления:

$$
\int_{\mathbb{R}_{+}} x^{(k)}(t) d t \rightarrow \max , \quad \int_{\mathbb{R}_{+}}\left(x^{2}(t)+\left(x^{(n)}(t)\right)^{2}\right) d t \leqslant 1 .
$$

2) Принцип Лагранжа. Функция Лагранжа здесь аналогична той, которая использовалась при решении задачи Габушина:

$$
\mathscr{L}=\int_{\mathbb{R}_{+}}\left(\left(x_{1}^{2}+u^{2}-\lambda x_{k+1}^{2}\right)+\sum_{i=1}^{n-1} p_{i}\left(\dot{x}_{i}-x_{i+1}\right)+p_{n}\left(\dot{x}_{n}-u\right)\right) d t .
$$

3) Исследование. Если $\widehat{x}(\cdot)$ - решение задачи (ii), то должно найтись такое число $\lambda>0$, что $\widehat{x}(\cdot)$ удовлетворяет уравнению Эйлера-Пуассона

$$
(-1)^{n} x^{(2 n)}+\lambda(-1)^{k+1} x^{(2 k)}+x=0 .
$$

Характеристический многочлен этого уравнения имеет вид:

$$
(-1)^{n} z^{2 n}+\lambda(-1)^{k+1} z^{2 k}+1=0
$$

и при малых $\lambda$ не имеет мнимых корней. Следовательно, он разлагается на два сомножителя, один из которых $z^{n}+\sum_{j=1}^{n} \nu_{j} z^{n-j}$ имеет корни в левой полуплоскости, и значит, $\widehat{x}(\cdot)$ удовлетворяет уравнению

$$
x^{(n)}+\sum_{j=1}^{n} \nu_{j} x^{(n-j)}=0 .
$$

После интегрирования по частям приходим к равенству

$$
\begin{aligned}
\int_{\mathbb{R}_{+}}\left(x^{(n)}+\sum_{j=1}^{n} \nu_{j} x^{(n-j)}\right)^{2} d t & \\
& =\int_{\mathbb{R}_{+}}\left(x^{2}-\lambda\left(x^{(k)}\right)^{2}+\left(x^{(n)}\right)^{2}\right) d t-Q_{\lambda}\left(x(0), \dot{x}(0), \ldots, x^{(n-1)}(0)\right),
\end{aligned}
$$

где $Q_{\lambda}$ - квадратичная форма. Так как для любого ненулевого вектора $\xi=\left(\xi_{1}, \ldots, \xi_{n}\right) \in \mathbb{R}^{n}$ сушествует ненулевое решение уравнения (а) такое, что $x^{(i-1)}(0)=\xi_{i}, i=1, \ldots, n$, то из (iii) вытекает, что $Q(\cdot)$ - положительно определенная форма (при малых $\lambda>0$ ). Следовательно, сушествует такое $\widehat{\lambda}$, что $Q_{\widehat{\lambda}}$ неотрицательна и обрашается в нуль на ненулевом векторе. Из (iii) следует неравенство

$$
\lambda \int_{\mathbb{R}_{+}}\left(x^{(k)}(t)\right)^{2} d t \leqslant \int_{\mathbb{R}_{+}}\left(x^{2}(t)+\left(x^{(n)}(t)\right)^{2}\right) d t,
$$

которое является точньм при $\lambda=\widehat{\lambda}$. Из этого точного неравенства уже стандартным образом выводится точное мультипликативное неравенство.

На этом первый параграф̆ закончен. Второй параграф, как уже говорилось, посвящен различным обобщениям рассмотренных задач (другие многообразия, дробные производные и т. п.). 


\section{$\S 2$.}

ЗАДАчА Іа (обшая задача Харди-Литтлвуда-Полиа на многообразии $\mathbb{S}^{d}$ ).

$$
\left\|(-\Delta)^{\alpha^{0} / 2} x(\cdot)\right\|_{L_{2}\left(\mathbb{S}^{d}\right)} \rightarrow \max , \quad\left\|(-\Delta)^{\alpha^{j} / 2} x(\cdot)\right\|_{L_{2}\left(\mathbb{S}^{d}\right)} \leqslant \gamma_{j}, \quad j=1, \ldots, N .
$$

Здесь $\alpha^{0} \geqslant 0, \alpha^{j} \geqslant 0, \gamma_{j}>0, j=1, \ldots, N, u(-\Delta)^{\alpha / 2}$ обозначает $\alpha / 2$-степень оператора Бельтрами-Лапласа на сфере $\mathbb{S}^{d}=\left\{x \in \mathbb{R}^{d+1}:\|x\|=1\right\}$.

Дадим некоторые пояснения относительно введенных понятий. Сфера $\mathbb{S}^{d}$ есть однородное пространство (относительно действия группы ортогональных матриц $O(n+1))$, на которой имеется единственная инвариантная относительно действия этой группы вероятностная мера Хаара и единственный (с точностью до множителя) инвариантный оператор второго порядка $-\Delta$, который называется оператором Бельтрами-Лапласа и который представляет собой “сферическую часть” оператора $\sum_{i=1}^{d+1} \partial^{2} / \partial t_{i}^{2}$ (см. [21]). Оператор Бельтрами-Лапласа положительный самосопряженный неограниченный оператор в $L_{2}\left(\mathbb{S}^{d}\right)$, собственные функции которого (после нормировки) образуют ортонормированный базис в $L_{2}\left(\mathbb{S}^{d}\right)$ и обычно называются сферическими гармониками. Обозначим их (исключая константу, поскольку далее мы рассматриваем только функции в среднем равные нулю) через $Y_{k j d}(\cdot)$, где $k \in \mathbb{N}, j=1, \ldots, \delta_{k d}$ и $\delta_{k d}=\frac{2 k+d-1}{k}\left(\begin{array}{c}k+d-2 \\ k-1\end{array}\right)$ (при $d=1$, т.е. в случае окружности, эти функции суть $\sin k \cdot$ и $\cos k \cdot)$. Для каждого $k \in \mathbb{N}$ функции $Y_{k j d}(\cdot), j=1, \ldots, \delta_{k d}$, отвечают собственному значению $\Lambda_{k d}=k(k+d-1)$ оператора $-\Delta$. Таким образом, если функция $x(\cdot) \in L_{2}\left(\mathbb{S}^{d}\right)$ и в среднем равна нулю, то

$$
x(\cdot)=\sum_{k \in \mathbb{N}} \sum_{j=1}^{\delta_{k d}} x_{k j} Y_{k j d}(\cdot)
$$

и

$$
(-\Delta) x(\cdot)=\sum_{k \in \mathbb{N}} \Lambda_{k d} \sum_{j=1}^{\delta_{k d}} x_{k j} Y_{k j d}(\cdot) .
$$

Для каждого $\alpha \in \mathbb{R}$ оператор $(-\Delta)^{\alpha / 2}$ определяется по правилу

$$
(-\Delta)^{\alpha / 2} x(\cdot)=\sum_{k \in \mathbb{N}} \Lambda_{k d}^{\alpha / 2} \sum_{j=1}^{\delta_{k d}} x_{k j} Y_{k j d}(\cdot) .
$$

Отметим, что случай $d=1$ рассматривался в [22].

РешениЕ ЗАДАчИ Іа. 1) Формализация. Так как

$$
\left\|(-\Delta)^{\alpha / 2} x(\cdot)\right\|_{L_{2}\left(\mathbb{S}^{d}\right)}^{2}=\sum_{k \in \mathbb{N}} \Lambda_{k d}^{\alpha / 2} \sum_{j=1}^{\delta_{k d}} x_{k j}^{2}
$$


то обозначая $g(k)=k(k+d-1)$ и $u_{k}=\sum_{j=1}^{\delta_{k d}} x_{k j}^{2}$, получаем, что задача (i) (с точностью до изменения знака ее значения) равносильна следующей:

$$
\begin{gathered}
-\sum_{k \in \mathbb{N}} g^{\alpha^{0}}(k) u_{k} \rightarrow \min \\
\sum_{k \in \mathbb{N}} g^{\alpha^{j}}(k) u_{k} \leqslant \gamma_{j}^{2}, \quad j=1, \ldots, N, \quad u_{k} \geqslant 0 \quad \forall k \in \mathbb{N} .
\end{gathered}
$$

Для решения этой задачи (линейного программирования) можно воспользоваться методами двойственности (как было сделано для ее аналога на $\mathbb{R}^{d}$ ). Однако, ради разнообразия, применим здесь принцип Лагранжа.

2) Принцип Лагранжа. Если последовательность $\left\{\widehat{u}_{k}\right\}_{k \in \mathbb{N}}-$ решение (ii), то найдутся такие множители Лагранжа $\lambda_{j} \geqslant 0, j=1, \ldots, N$, что для всех $\left\{u_{k}\right\}_{k \in \mathbb{N}}$ таких, что $u_{k} \geqslant 0, k \in \mathbb{N}$, выполняются неравенство (мы не выписываем функцию Лагранжа в виду ее очевидности)

$$
\sum_{k \in \mathbb{N}}\left(-g^{\alpha^{0}}(k)+\sum_{j=1}^{N} \lambda_{j} g^{\alpha^{j}}(k)\right) u_{k} \geqslant \sum_{k \in \mathbb{N}}\left(-g^{\alpha^{0}}(k)+\sum_{j=1}^{N} \lambda_{j} g^{\alpha^{j}}(k)\right) \widehat{u}_{k}
$$

и условия дополняющей нежесткости

$$
\lambda_{j}\left(\sum_{k \in \mathbb{N}} g^{\alpha^{j}}(k) \widehat{u}_{k}-\gamma_{j}^{2}\right)=0, \quad j=1, \ldots, N
$$

3) Исследование. Покажем сначала, что для любых $\lambda_{j} \geqslant 0, j=1, \ldots, N$, функция

$$
l(k)=-g^{\alpha^{0}}(k)+\sum_{j=1}^{N} \lambda_{j} g^{\alpha^{j}}(k)
$$

может иметь не более двух нулей на $\mathbb{N}$. Рассмотрим для этого на $\mathbb{R}_{+}$функцию

$$
l_{1}(t)=-g_{1}^{\alpha^{0}}(t)+\sum_{j=1}^{N} \lambda_{j} g_{1}^{\alpha^{j}}(t)
$$

где $g_{1}(t)=t(t+d-1)$, и на $\mathbb{R}$ функцию

$f(\tau)=-\exp \left(\alpha^{0} \tau\right)+\sum_{j=1}^{N} \lambda_{j} \exp \left(\alpha^{j} \tau\right)=-\exp \left(\alpha^{0} \tau\right)\left(-1+\sum_{j=1}^{N} \lambda_{j} \exp \left(\left(\alpha^{j}-\alpha^{0}\right) \tau\right)\right)$

Функция в скобках строго выпукла и поэтому не может иметь более двух нулей. Следовательно, функция $f(\cdot)$ имеет не более двух нулей. Но тогда и $l_{1}(\cdot)$ имеет не более двух нулей на $(0, \infty)$. В самом деле, пусть $0<t_{1}<t_{2}<t_{3}-$ нули $l_{1}(\cdot)$. Положим $\tau_{i}=\ln g_{1}\left(t_{i}\right), i=1,2,3$. Так как $g_{1}(\cdot)$ строго монотонно возрастает, то $\tau_{1}<\tau_{2}<\tau_{3}$ и при этом, очевидно, $f\left(\tau_{i}\right)=0, i=1,2,3$, что невозможно. Итак, 
функция $l(\cdot)$ зануляется не более чем в двух точках. Если $\alpha^{0}<\alpha^{j}, j=1, \ldots, N$, то ясно, что $l(\cdot)$ может иметь лишш один нуль.

Отметим далее, что если задача (ii) имеет решение, то легко проверяется, что функция $l(\cdot)\left(\right.$ с соответствующими $\left.\lambda_{j}\right)$ неотрицательна, и значит, если $k_{1}$ и $k_{2}-\mathrm{ee}$ нули, то $k_{2}=k_{1}+1$, а само решение сосредоточено в нулях $l(\cdot)$. Если же $\alpha^{0}<\alpha^{j}$, $j=1, \ldots, N$, то единственный нуль $l(\cdot)$ может быть только при $k=1$, так как иначе $l(\cdot)$ принимала бы отрицательные значения.

Найдем сначала решение задачи (ii) в важном частном случае, когда $N=2$. При этом, очевидно, можно считать, что $0 \leqslant \alpha^{1}<\alpha^{2}$ и $\alpha^{0} \neq \alpha^{j}, j=1,2$. Рассмотрим следуюшие уравнения:

$$
\lambda_{1} g^{\alpha^{1}}(k)+\lambda_{2} g^{\alpha^{2}}(k)=g^{\alpha^{0}}(k), \quad \lambda_{1} g^{\alpha^{1}}(k+1)+\lambda_{2} g^{\alpha^{2}}(k+1)=g^{\alpha^{0}}(k+1)
$$

и

$$
\lambda_{j}\left(g^{\alpha^{1}}(k) u+g^{\alpha^{2}}(k+1) v-\gamma_{j}^{2}\right)=0, \quad j=1,2,
$$

относительно неизвестных $\lambda_{1}, \lambda_{2}, u, v$ и $k$.

Пусть $\alpha^{0}<\alpha^{1}$ и $\gamma_{2}^{2} / \gamma_{1}^{2}<g^{\alpha^{2}-\alpha^{1}}(1)=d^{\alpha^{2}-\alpha^{1}}$. Положим $k=1, u=\gamma_{2}^{2} d^{-\alpha^{2}}$, $v=0, \lambda_{1}=0$ и $\lambda_{2}=d^{\alpha^{0}-\alpha^{2}}$. Тогда первое уравнение в (v) выполняется и оно означает, что $l(1)=0$. Второе выполняется поскольку функция $l(\cdot)$ может иметь лишь один нуль на $\mathbb{N}$ (см. выше). Соотношения (vi) выполнены очевидным образом.

Рассмотрим теперь последовательность $\left\{\widehat{u}_{k}\right\}_{k \in \mathbb{N}}$, где $\widehat{u}_{1}=\gamma_{2}^{2} d^{-\alpha^{2}}$ и $\widehat{u}_{k}=0$, если $k \geqslant 2$. Элементарно проверяется, что это допустимая последовательность в (ii) (при $N=2$ ). Тогда из соотношений (v), (vi) и условий достаточности в задачах вьпуклого программирования легко следует, что $\left\{\widehat{u}_{k}\right\}_{k \in \mathbb{N}}-$ решение задачи (ii) и ее значение равно $-\sum_{k \in \mathbb{N}} g^{\alpha^{0}}(k) \widehat{u}_{k}=-\gamma_{2}^{2} d^{\alpha^{0}-\alpha^{2}}$.

Схема дальнейших рассуждений такая же и поэтому будем более кратки. Пусть по-прежнему $\alpha^{0}<\alpha^{1}$. Если $\gamma_{2}^{2} / \gamma_{1}^{2}=d^{\alpha^{2}-\alpha^{1}}$, то полагая в (v) и (vi) $k=1$, $u=\gamma_{2}^{2} d^{-\alpha^{2}}\left(=\gamma_{1}^{2} d^{-\alpha^{1}}\right), v=0$ и выбирая любые неотрицательные $\lambda_{1}$ и $\lambda_{2}$, удовлетворяюшие (v), получаем, что значение задачи в данном случае также равно $-\gamma_{2}^{2} d^{\alpha^{0}-\alpha^{2}}\left(=-\gamma_{1}^{2} d^{\alpha^{0}-\alpha^{1}}\right)$. Если же $\gamma_{2}^{2} / \gamma_{1}^{2}>d^{\alpha^{2}-\alpha^{1}}$, то полагая в (v) и (vi) $k=1, u=\gamma_{1}^{2} d^{-\alpha^{1}}, v=0, \lambda_{1}=d^{\alpha^{0}-\alpha^{1}}$ и $\lambda_{2}=0$, получаем, что значение задачи равно $-\gamma_{1}^{2} d^{\alpha^{0}-\alpha^{1}}$.

Эти результаты можно объединить в одну формулу, а именно, если $\alpha^{0}<\alpha^{1}$, то значение задачи (ii) $(N=2)$ есть $-\min \left\{\gamma_{1}^{2} d^{\alpha^{0}-\alpha^{1}}, \gamma_{2}^{2} d^{\alpha^{0}-\alpha^{2}}\right\}$.

Пусть теперь $\alpha^{1}<\alpha^{0}<\alpha^{2}$. Если $\gamma_{2}^{2} / \gamma_{1}^{2} \leqslant d^{\alpha^{2}-\alpha^{1}}$, то, как и выше, полагая в (v) и (vi) $k=1, u=\gamma_{2}^{2} d^{-\alpha^{2}}, v=0, \lambda_{1}=0, \lambda_{2}=d^{\alpha^{0}-\alpha^{2}}$, получаем, что значение задачи равно $-\gamma_{2}^{2} d^{\alpha^{0}-\alpha^{2}}$. Если же $\gamma_{2}^{2} / \gamma_{1}^{2}>d^{\alpha^{2}-\alpha^{1}}$, то рассмотрим функцию $G(t)=(t(t+d-1))^{\alpha^{2}-\alpha^{1}}, t \geqslant 0$, и положим $\widehat{k}=\left[G^{-1}\left(\gamma_{2}^{2} / \gamma_{1}^{2}\right)\right]$. Пусть $\lambda_{1}$ и $\lambda_{2}-$ решение системы (v) при $k=\widehat{k}$. Очевидно, это решение единственно и его компоненты положительны (и то, и другое есть следствие только того, что $\alpha^{1}<\alpha^{0}<\alpha^{2}$ ). Тогда соотношения (vi) преврашаются (для каждого $k \in \mathbb{N}$ ) в систему двух уравнений относительно неизвестных $u$ и $v$. Эта система всегда имеет единственное решение, но, как следует из правила Крамера, компоненты решения неотрицательны тогда и только тогда, когда $k=\widehat{k}$. Обозначим их, соответственно, 
$u(\widehat{k})$ и $v(\widehat{k})$. Тогда, как и выше, проверяется (используя достаточные условия), что последовательность $\left\{\widehat{u}_{k}\right\}_{k \in \mathbb{N}}$, где $\widehat{u}_{\widehat{k}}=u(\widehat{k}), \widehat{u}_{\widehat{k}+1}=v(\widehat{k})$ и $\widehat{u}_{k}=0$, если $k \neq \widehat{k}, \widehat{k}+1$, является решением задачи (ii) $(N=2)$ в рассматриваемом случае. Находя явные выражения для $u(\widehat{k})$ и $v(\widehat{k})$ и для любых $\alpha$ и $\beta$ обозначая $\kappa(\alpha, \beta)=g^{\alpha}(\widehat{k}) g^{\beta}(\widehat{k}+1)-g^{\beta}(\widehat{k}) g^{\alpha}(\widehat{k}+1)$, получаем, что значение задачи равно

$$
-\left(\gamma_{1}^{2} \kappa\left(\alpha^{0}, \alpha^{2}\right)+\gamma_{2}^{2} \kappa\left(\alpha^{1}, \alpha^{0}\right)\right)\left(\kappa\left(\alpha^{1}, \alpha^{2}\right)\right)^{-1} .
$$

Пусть $\alpha^{0}>\alpha^{2}$. Тогда значение задачи равно $-\infty$. Действительно, для каждого $m \in \mathbb{N}$ рассмотрим последовательность $\left\{u_{k}^{m}\right\}_{k \in \mathbb{N}}$, где $u_{m}^{m}=\gamma_{2}^{2} / g^{\alpha^{2}}(m)$ и $u_{k}^{m}=0$, если $k \neq m$. Легко видеть, что для достаточно больших $m$ это допустимые последовательности и $-\sum_{k \in \mathbb{N}} g^{\alpha^{0}}(k) u_{k}^{m}=-\gamma_{2}^{2} g^{\alpha^{0}-\alpha^{2}}(m) \rightarrow-\infty$ при $m \rightarrow \infty$.

Объединяя полученные результаты, приходим к тому, что значение задачи Іа при $N=2$ имеет вид:

$$
\begin{aligned}
& S_{\mathrm{Ia}}\left(\alpha^{0}, \alpha^{1}, \alpha^{2}, \gamma_{1}, \gamma_{2}\right) \\
& \quad= \begin{cases}\min \left\{\gamma_{1} d^{\left(\alpha^{0}-\alpha^{1}\right) / 2}, \gamma_{2} d^{\left(\alpha^{0}-\alpha^{2}\right) / 2}\right\}, & \alpha^{0}<\alpha^{1}, \\
\sqrt{\gamma_{1}^{2} \frac{\kappa\left(\alpha^{0}, \alpha^{2}\right)}{\kappa\left(\alpha^{1}, \alpha^{2}\right)}+\gamma_{2}^{2} \frac{\kappa\left(\alpha^{1}, \alpha^{0}\right)}{\kappa\left(\alpha^{1}, \alpha^{2}\right)}}, & \alpha^{1}<\alpha^{0}<\alpha^{2}, \frac{\gamma_{2}}{\gamma_{1}}>d^{\left(\alpha^{2}-\alpha^{1}\right) / 2}, \\
\gamma_{2} d^{\left(\alpha^{0}-\alpha^{2}\right) / 2}, & \alpha^{1}<\alpha^{0}<\alpha^{2}, \frac{\gamma_{2}}{\gamma_{1}} \leqslant d^{\left(\alpha^{2}-\alpha^{1}\right) / 2}, \\
+\infty, & \alpha^{0}>\alpha^{2} .\end{cases}
\end{aligned}
$$

Отсюда уже нетрудно получить значение задачи для любого $N \geqslant 2$.

Teоpema Іа. Пусть в задаче Ia $\alpha^{0}, \alpha^{j} \in \mathbb{R}_{+}^{d}, \gamma_{j}>0, j=1, \ldots, N, u$ $S_{\mathrm{Ia}}=S_{\mathrm{Ia}}\left(\alpha^{0}, \alpha^{1}, \ldots, \alpha^{N}, \gamma_{1}, \ldots, \gamma_{N}\right)-$ ее значение. Тогда

1) если $\alpha^{0} \leqslant \max _{1 \leqslant j \leqslant N} \alpha^{j}$, mo $S_{\mathrm{Ia}}=\min S_{\mathrm{Ia}}\left(\alpha^{0}, \alpha^{j_{1}}, \alpha^{j_{2}}, \gamma_{j_{1}}, \gamma_{j_{2}}\right)$, әде $\alpha^{j_{1}} \leqslant \alpha^{0} \leqslant \alpha^{j_{2}}$

2) если $\alpha^{0}>\max _{1 \leqslant j \leqslant N} \alpha^{j}$, mo $S_{\text {Ia }}=+\infty$.

Перейдем теперь к некоторым обобшениям задачи Габушина. Начнем с рассмотрения иного способа доказательства достаточности в исходной постановке, который позволяет исследовать и более общие задачи. Остановимся здесь на одной из них, а именно, на задаче

$$
\left\|\sum_{i=1}^{n} a_{i} x^{(n-i)}(\cdot)\right\|_{C^{b}\left(\mathbb{R}_{+}\right)} \rightarrow \max , \quad\|x(\cdot)\|_{L_{2}\left(\mathbb{R}_{+}\right)}^{2}+\left\|x^{(n)}(\cdot)\right\|_{L_{2}\left(\mathbb{R}_{+}\right)}^{2} \leqslant 1
$$

где $a_{i} \in \mathbb{R}, i=1, \ldots, n$.

В качестве формализации (i) рассмотрим задачу

$$
\sum_{i=1}^{n} a_{i} x^{(n-i)}(0) \rightarrow \max , \quad \int_{\mathbb{R}_{+}}\left(x^{2}(t)+\left(x^{(n)}(t)\right)^{2}\right) d t \leqslant 1 .
$$


Если $\widehat{x}(\cdot)$ - решение задачи (ii), то так же, как и раньше (см. доказательство неравенства Габушина), устанавливается, что $\widehat{x}(\cdot)$ удовлетворяет уравнению

$$
x^{(n)}+\sum_{j=1}^{n} \nu_{j} x^{(n-j)}=0
$$

После интегрирования по частям получаем, что

$$
\begin{aligned}
\int_{\mathbb{R}_{+}}\left(x^{(n)}(t)+\right. & \left.\sum_{j=1}^{n} \nu_{j} x^{(n-j)}(t)\right)^{2} d t \\
& =\int_{\mathbb{R}_{+}}\left(x^{2}(t)+\left(x^{(n)}(t)\right)^{2}\right) d t-Q\left(x(0), \dot{x}(0), \ldots, x^{(n-1)}(0)\right),
\end{aligned}
$$

где $Q(\cdot)$ - квадратичная форма, причем положительно определенная (см. соответствуюшее место в задаче Любича-Купцова).

Из (iii) следует, что

$$
Q\left(x(0), \dot{x}(0), \ldots, x^{(n-1)}(0)\right) \leqslant \int_{\mathbb{R}_{+}}\left(x^{2}(t)+\left(x^{(n)}(t)\right)^{2}\right) d t .
$$

Пусть $\widehat{\xi}=\left(\widehat{\xi}_{1}, \ldots, \widehat{\xi}_{n}\right)-$ точка минимума линейной формы $l: \xi \mapsto \sum_{i=1}^{n} a_{i} \xi_{i}$ на эллипсоиде $\left\{\xi \in \mathbb{R}^{n}: Q(\xi) \leqslant 1\right\}$. Ясно, что $\widehat{\xi}$-единственная точка минимума и что $Q(\widehat{\xi})=1$. Обозначим через $\widehat{x}(\cdot)$ решение уравнения (а) с начальными условиями $\widehat{x}^{(i-1)}(0)=\widehat{\xi}_{i}, i=1, \ldots, n$. Тогда из (iii) следует, что $\widehat{x}(\cdot)$ удовлетворяет ограничениям в задаче (ii). Покажем, что $\widehat{x}(\cdot)$ - решение этой задачи. Действительно, если существует такая допустимая функция $x(\cdot)$, что величина $\sum_{i=1}^{n} a_{i} x^{(n-i)}(0)$ больше $l(\widehat{\xi})$, то $Q\left(x(0), \dot{x}(0), \ldots, x^{(n-1)}(0)\right)>1$, а это противоречит неравенствy (iv).

Следуюшее обобщение результата Габушина связано с заменой целой промежуточной производной на дробную. Другими словами, мы рассматриваем задачу

$$
\left\|D^{\alpha} x(\cdot)\right\|_{C^{b}\left(\mathbb{R}_{+}\right)} \rightarrow \max , \quad\|x(\cdot)\|_{L_{2}\left(\mathbb{R}_{+}\right)} \leqslant \gamma_{1}, \quad\left\|x^{(n)}(\cdot)\right\|_{L_{2}\left(\mathbb{R}_{+}\right)} \leqslant \gamma_{2} .
$$

Здесь $D^{\alpha} x(\cdot)$ обозначает дробную производную порядка $\alpha \in \mathbb{R}$ функции $x(\cdot)$. Напомним, что (см., например, [23])

$$
D^{\alpha} x(\cdot):= \begin{cases}\frac{d^{[\alpha]+1}}{d t^{[\alpha]+1}} \circ I^{[\alpha]+1-\alpha} x(\cdot), & \alpha \geqslant 0, \\ I^{-\alpha} x(\cdot), & \alpha<0,\end{cases}
$$

где

$$
I^{\beta} x(t):=-\frac{1}{\Gamma(\beta)} \int_{t}^{\infty}(\tau-t)^{\beta-1} x(\tau) d \tau, \quad \beta>0,
$$

и $Г(\cdot)$ - гамма-функция Эйлера. Производная $D^{\alpha}$ назьвается дробной производной в смысле Римана-Лиувилля. 
Если $\alpha \geqslant 0$ и функция $x(\cdot)$ достаточно “хорошая” (гладкая и быстро убывает), то

$$
D^{\alpha} x(t)=-\frac{1}{\Gamma([\alpha]-\alpha+1)} \int_{\mathbb{R}_{+}} \tau^{[\alpha]-\alpha} x^{([\alpha]+1)}(\tau+t) d \tau, \quad t \geqslant 0 .
$$

Ясно, что, когда $\alpha=m \in \mathbb{Z}_{+}$, это выражение совпадает с обычной производной $x^{(m)}(t)$, а когда $-1 \leqslant \alpha<0$, оно равно $I^{-\alpha} x(t)$.

Рассмотрим случай $n=1$. В общей ситуации рассуждения (за исключением некоторых технических усложнений) остаются такими же. Итак, мы рассматриваем задачу

$$
\left\|D^{\alpha} x(\cdot)\right\|_{C^{b}\left(\mathbb{R}_{+}\right)} \rightarrow \max , \quad\|x(\cdot)\|_{L_{2}\left(\mathbb{R}_{+}\right)} \leqslant \delta, \quad\|\dot{x}(\cdot)\|_{L_{2}\left(\mathbb{R}_{+}\right)} \leqslant 1,
$$

где числа $\gamma_{1}=\delta$ и $\gamma_{2}=1$ выбраны из тех же соображений, что и раньше (см. задачу Габушина). Нетрудно проверить, что условие $\alpha \in\left(-\frac{1}{2}, \frac{1}{2}\right)$ необходимо для конечности задачи (i). Пусть $0<\alpha<\frac{1}{2}$.

РЕшениЕ. 1) Формализация. Естественной формализацией (i) является следующая задача:

$$
-\frac{1}{\Gamma(1-\alpha)} \int_{\mathbb{R}_{+}} t^{-\alpha} \dot{x}(t) d t \rightarrow \min , \quad \int_{\mathbb{R}_{+}} x^{2}(t) d t \leqslant \delta^{2}, \int_{\mathbb{R}_{+}} \dot{x}^{2}(t) d t \leqslant 1 .
$$

2) Принцип Лагранжа. Задача (ii) - задача вариационного исчисления и одновременно задача выпуклого программирования. Ее функция Лагранжа:

$\mathscr{L}=-\lambda_{0}(\Gamma(1-\alpha))^{-1} \int_{\mathbb{R}_{+}} t^{-\alpha} \dot{x}(t) d t+\lambda_{1}\left(\int_{\mathbb{R}_{+}} x^{2}(t) d t-\delta^{2}\right)+\lambda_{2}\left(\int_{\mathbb{R}_{+}} \dot{x}^{2}(t) d t-1\right)$.

Если $\widehat{x}(\cdot)$ - решение задачи, то найдутся такие $\lambda_{1} \geqslant 0$ и $\lambda_{2} \geqslant 0$ (можно считать $\left.\lambda_{0}=1\right)$, что $\widehat{x}(\cdot)$ удовлетворяет уравнению Эйлера

$$
\lambda_{2} \ddot{x}-\lambda_{1} x=(2 \Gamma(-\alpha))^{-1} t^{-\alpha-1},
$$

условиям трансверсальности

$$
\lim _{\substack{t \rightarrow 0+\\ t \rightarrow \infty}}\left(2 \lambda_{2} \dot{x}(t)-(\Gamma(1-\alpha))^{-1} t^{-\alpha}\right)=0
$$

и условиям дополняющей нежесткости

$$
\lambda_{1}\left(\int_{\mathbb{R}_{+}} x^{2}(t) d t-\delta^{2}\right)=0, \quad \lambda_{2}\left(\int_{\mathbb{R}_{+}} \dot{x}^{2}(t) d t-1\right)=0 .
$$

3) Исследование. Решая (а) методом вариации произвольных постоянных и используя (b) и (c) для нахождения всех констант, получаем, что если $\widehat{x}(\cdot)$ - решение (ii), то оно имеет вид:

$$
\widehat{x}(t)=-\left(\frac{\left(\lambda_{1} / \lambda_{2}\right)^{(\alpha-1) / 2}}{4 \lambda_{2}}\right) x_{0}\left(\left(\frac{\lambda_{1}}{\lambda_{2}}\right)^{1 / 2} t\right),
$$


где

$$
x_{0}(t)=\left(e^{-t}+e^{t}-\frac{e^{-t}}{\Gamma(1-\alpha)} \int_{0}^{t} \tau^{-\alpha} e^{\tau} d \tau-\frac{e^{t}}{\Gamma(1-\alpha)} \int_{0}^{t} \tau^{-\alpha} e^{-\tau} d \tau\right),
$$

$\lambda_{1}=(1 / 4)(A / B)^{(1+2 \alpha) / 2} A \delta^{-(3+2 \alpha) / 2}, \quad \lambda_{2}=(1 / 4)(B / A)^{(1-2 \alpha) / 2} B \delta^{(1-2 \alpha) / 2}$ и $A=\left\|x_{0}(\cdot)\right\|_{L_{2}\left(\mathbb{R}_{+}\right)}, B=\left\|\dot{x}_{0}(\cdot)\right\|_{L_{2}\left(\mathbb{R}_{+}\right)}$(элементарная проверка показывает, что $\left.x_{0}(\cdot) \in \mathscr{W}_{2}^{1}\left(\mathbb{R}_{+}\right)\right)$.

Условие минимума функции Лагранжа в точке $\widehat{x}(\cdot)$ равносильно выполнению для всех $x(\cdot) \in \mathscr{W}_{2}^{1}\left(\mathbb{R}_{+}\right)$следующего равенства:

$$
-\frac{1}{\Gamma(1-\alpha)} \int_{\mathbb{R}_{+}} t^{-\alpha} \dot{x}(t) d t=2 \lambda_{1} \int_{\mathbb{R}_{+}} \widehat{x}(t) x(t) d t+2 \lambda_{2} \int_{\mathbb{R}_{+}} \dot{\hat{x}}(t) \dot{x}(t) d t,
$$

из которого, как и ранњше, следует решение трех задач. Введем предварительно обозначение

$$
K(\alpha)=\frac{1}{2}\left(\frac{A}{B}\right)^{\alpha} \frac{A^{2}+B^{2}}{\sqrt{A B}} .
$$

А) Неравенство для производных. Проводя те же оценки, что и ранњше (см. задачу Габушина при $n=1$ ), и учитывая тот (элементарно проверяемьй факт), что если $x(\cdot) \in \mathscr{W}_{2}^{1}\left(\mathbb{R}_{+}\right)$, то в определении дробной производной интеграл и производную можно поменять местами, получаем из (iii) неравенство $\left|D^{\alpha}(0)\right| \leqslant$ $K(\alpha) \delta^{(1-2 \alpha) / 2}$, которое на $\widehat{x}(\cdot)$ обрашается в равенство. Отсюда следует, что $\widehat{x}(\cdot)$ - решение задачи (ii). Из этого же неравенства и из соображений однородности следует точное мультипликативное неравенство

$$
\left\|D^{\alpha} x(\cdot)\right\|_{C^{b}\left(\mathbb{R}_{+}\right)} \leqslant K(\alpha)\|x(\cdot)\|_{L_{2}\left(\mathbb{R}_{+}\right)}^{(1-2 \alpha) / 2}\|\dot{x}(\cdot)\|_{L_{2}\left(\mathbb{R}_{+}\right)}^{(1+2 \alpha) / 2}
$$

и, таким образом, $K_{\mathbb{R}_{+}}(1, \alpha, 2, \infty, 2)=K(\alpha)$.

В) Оптимальный метод восстановления функционала $x(\cdot) \mapsto D^{\alpha}(0)$ на классе $W_{2}^{1}\left(\mathbb{R}_{+}\right)$при условии, что $x(\cdot)$ в $L_{2}\left(\mathbb{R}_{+}\right)$известна с точностью до $\delta$, задается формулой

$$
D^{\alpha} x(0) \approx 2 \lambda_{1} \int_{\mathbb{R}_{+}} \widehat{x}(t) y(t) d t
$$

где $\lambda_{1}$ определено вьшше, и при этом

$$
e\left(D^{\alpha} x(0), W_{2}^{1}\left(\mathbb{R}_{+}\right), \delta\right)=K(\alpha) \delta^{(1-2 \alpha) / 2}
$$

Проверяются эти утверждения дословно так же, как и раньше в задаче Габушина.

С) Пусть $N>0$ и $\delta$ выбрано из условия $2 \lambda_{1} \delta=N$. Тогда функционал $x^{*}$, сопоставляющий $x(\cdot) \in L_{2}\left(\mathbb{R}_{+}\right)$первый интеграл в (iii), имеет норму $N$ и так же, как и ранњше, проверяется, что он осушествляет наилучшее приближение функционала $x(\cdot) \mapsto D^{\alpha} x(0)$ на классе $W_{2}^{1}\left(\mathbb{R}_{+}\right)$и при этом

$$
E\left(N, D^{\alpha} x(0), W_{2}^{1}\left(\mathbb{R}_{+}\right)\right)=\frac{B}{2}\left(\frac{A}{2 N}\right)^{\frac{1-2 \alpha}{1+2 \alpha}} .
$$

Общий случай, т.е. когда $n \in \mathbb{N}$ произвольно, рассматривается, как уже говорилось, по сути дела так же. Мы ограничимся здесь задачей о вычислении точной константы в соответствующем неравенстве для дробной производной. 
Tеорема. Неравенство

$$
\left\|D^{\alpha} x(\cdot)\right\|_{C^{b}\left(\mathbb{R}_{+}\right)} \leqslant K\|x(\cdot)\|_{L_{2}\left(\mathbb{R}_{+}\right)}^{\nu_{1}}\left\|x^{(n)}(\cdot)\right\|_{L_{2}\left(\mathbb{R}_{+}\right)}^{\nu_{2}}
$$

с положительньми $\nu_{1}$ и $\nu_{2}$ имеет место для всех функций $x(\cdot) \in W_{2}^{n}\left(\mathbb{R}_{+}\right)$ тогда и только тогда, когда

$$
-\frac{1}{2}<\alpha<n-\frac{1}{2}, \quad \nu_{1}=\frac{1}{n}\left(n-\alpha-\frac{1}{2}\right), \quad \nu_{2}=1-\nu_{1} .
$$

Экстремальная функчия в (а) имеет вид:

$$
\begin{aligned}
x_{0}(t)=\frac{1}{\Gamma([\alpha]-\alpha+1)}\left(\sum_{j=1}^{2 n} a_{j} e^{\beta_{j} t} \int_{0}^{t} \tau^{[\alpha]-\alpha} e^{-\beta_{j} \tau} d \tau\right. \\
\left.\quad-\sum_{j=n+1}^{2 n} a_{j} e^{\beta_{j} t} \int_{\mathbb{R}_{+}} \tau^{[\alpha]-\alpha} e^{-\beta_{j} \tau} d \tau+\sum_{j=1}^{n} b_{j} e^{\beta_{j} t}\right),
\end{aligned}
$$

где $\beta_{1}, \ldots, \beta_{2 n}-$ корни уравнения $z^{2 n}+(-1)^{n}=0\left(\operatorname{Re} \beta_{j}<0, j=1, \ldots, n\right)$, $a_{1}, \ldots, a_{2 n}$ - единственное решение системы уравнений

$$
\sum_{j=1}^{2 n} \beta_{j}^{k} \xi_{j}= \begin{cases}(-1)^{n-[\alpha]}, & k=2 n-[\alpha]-2, \\ 0, & k=0,1, \ldots, 2 n-1, k \neq 2 n-[\alpha]-2,\end{cases}
$$

$u b_{1}, \ldots, b_{n}-$ единственное решение системы уравнений

$$
\sum_{j=1}^{n} \beta_{j}^{k} \eta_{j}=\sum_{j=n+1}^{2 n} a_{j} \beta_{j}^{k} \int_{\mathbb{R}_{+}} \tau^{[\alpha]-\alpha} e^{-\beta_{j} \tau} d \tau, \quad k=n, \ldots, 2 n-1 .
$$

Для наилучшей константы в (а) справедливо выражсение

$$
K_{\mathbb{R}_{+}}(n, \alpha, 2, \infty, 2)=\left(\frac{2 n}{2(n-\alpha)-1}\right)^{\frac{2(n-\alpha)-1}{4 n}}\left(\frac{2 n}{2 n-1}\right)^{\frac{2(\alpha+1)}{4 n}}\left\|x_{0}(\cdot)\right\|_{\mathscr{W}_{2}^{n}\left(\mathbb{R}_{+}\right)},
$$

¿де $\|x(\cdot)\|_{\mathscr{W}_{2}^{n}\left(\mathbb{R}_{+}\right)}=\|x(\cdot)\|_{L_{2}\left(\mathbb{R}_{+}\right)}+\left\|x^{(n)}(\cdot)\right\|_{L_{2}\left(\mathbb{R}_{+}\right)}$.

По поводу этого результата см. также [24].

Следуюшая постановка представляет собой “смесь" задач Тайкова и Габушина. Она заключается в оценке производной в метрике $C^{b}\left(\mathbb{R}_{+} \times \mathbb{R}^{d-1}\right)$ через производные в метрике $L_{2}\left(\mathbb{R}_{+} \times \mathbb{R}^{d-1}\right)$. Чтобы не выписывать громоздкие формулы, ограничимся простым модельным примером, когда $d=2$.

ЗАДАчА IIІа (об оценке производной в метрике $C^{b}\left(\mathbb{R}_{+} \times \mathbb{R}\right)$ через производные в метрике $\left.L_{2}\left(\mathbb{R}_{+} \times \mathbb{R}\right)\right)$. Рассмотрим задачу $\left((t, \tau) \in \mathbb{R}_{+} \times \mathbb{R}\right)$

$$
\begin{gathered}
\|x(\cdot, \cdot)\|_{C^{b}\left(\mathbb{R}_{+} \times \mathbb{R}\right)} \rightarrow \max , \\
\|x(\cdot, \cdot)\|_{L_{2}\left(\mathbb{R}_{+} \times \mathbb{R}\right)} \leqslant 1,\left\|\frac{\partial x(\cdot, \cdot)}{\partial t}\right\|_{L_{2}\left(\mathbb{R}_{+} \times \mathbb{R}\right)} \leqslant 1,\left\|\frac{\partial^{2} x(\cdot, \cdot)}{\partial \tau^{2}}\right\|_{L_{2}\left(\mathbb{R}_{+} \times \mathbb{R}\right)} \leqslant 1 .
\end{gathered}
$$


РЕшЕниЕ. Задача редуцируется к следующей проблеме вариационного исчисления:

$$
\int_{\mathbb{R}_{+} \times \mathbb{R}}\left(x^{2}(t, \tau)+\left(\frac{\partial x(t, \tau)}{\partial t}\right)^{2}+\left(\frac{\partial^{2} x(t, \tau)}{\partial \tau^{2}}\right)^{2}\right) d t d \tau \rightarrow \min , \quad x(0,0)=1
$$

Обозначим через $y(t, \sigma)$ преобразование Фурье функции $x(t, \tau)$ по $\tau$ и, используя равенство Парсеваля, получим, что задача (ii) равносильна следующей:

$$
\frac{1}{2 \pi} \int_{\mathbb{R}_{+} \times \mathbb{R}}\left(\left(1+\sigma^{4}\right) y^{2}(t, \sigma)+y_{t}^{\prime 2}(t, \sigma)\right) d t d \sigma \rightarrow \min , \quad \frac{1}{2 \pi} \int_{\mathbb{R}} y(0, \sigma) d \sigma=1
$$

Пусть $z(\cdot)$ - произвольная функция из $L_{1}(\mathbb{R})$. Для каждого $\sigma \in \mathbb{R}$ рассмотрим задачу

$$
\int_{\mathbb{R}_{+}}\left(\left(1+\sigma^{4}\right) y^{2}(t, \sigma)+y_{t}^{\prime 2}(t, \sigma)\right) d t \rightarrow \min , \quad y(0, \sigma)=z(\sigma),
$$

которая есть, фактически, задача Габушина для $n=1$ и $\alpha=0$ (последняя получается из (iv) при $\sigma=0$ и $z(\sigma)=1$ ). Поэтому, поступая точно так же, как и ранњше, получаем, что функция $y(t, \sigma)=z(\sigma) \exp \left(-t \sqrt{1+\sigma^{4}}\right)-$ решение задачи (iv). Подставляя эту функцию в (iii), приходим к задаче

$$
\frac{1}{2 \pi} \int_{\mathbb{R}} z^{2}(\sigma) \sqrt{1+\sigma^{4}} d \sigma \rightarrow \min , \quad \frac{1}{2 \pi} \int_{\mathbb{R}} z(\sigma) d \sigma=1
$$

По неравенству Коши-Буняковского

$$
1=\frac{1}{2 \pi} \int_{\mathbb{R}} z(\sigma) d \sigma \leqslant\left(\frac{1}{2 \pi} \int_{\mathbb{R}} z^{2}(\sigma) \sqrt{1+\sigma^{4}} d \sigma\right)^{1 / 2}\left(\frac{1}{2 \pi} \int_{\mathbb{R}} \frac{d \sigma}{\sqrt{1+\sigma^{4}}}\right)^{1 / 2}
$$

следует, что $a=2 \pi\left(\int_{\mathbb{R}}\left(1+\sigma^{4}\right)^{-1 / 2} d \sigma\right)^{-1}$ - значение задачи (iii') и, тем самым, - задачи (ii), функция $z(\sigma)=a / \sqrt{1+\sigma^{4}}-$ решение задачи (iii' ${ }^{\prime}$ и, значит, функция

$$
x(t, \tau)=\left(\int_{\mathbb{R}} \frac{d \sigma}{\sqrt{1+\sigma^{4}}}\right)^{-1} \int_{\mathbb{R}} \frac{e^{-t \sqrt{1+\sigma^{4}}}}{\sqrt{1+\sigma^{4}}} e^{i \tau \sigma} d \sigma
$$

есть решение задачи (ii).

Редукция задачи (ii) к задаче (i) осушествляется стандартным образом; рассмотрение более общей ситуации происходит по такой же схеме, что и в разобранном примере, поэтому более подробно на этих вопросах останавливаться здесь не будем. 
Неравенство Колмогорова. Как уже было сказано, в 1939 году Колмогоров [3] доказал точное неравенство (1) для любых $k, n \in \mathbb{N}$, когда $p=q=r=\infty$, $T=\mathbb{R}$, и при этом

$$
K_{\mathbb{R}}(n, k, \infty, \infty, \infty)=K_{n-k} / K_{n}^{(k-n) / n},
$$

где

$$
K_{r}=\frac{4}{\pi} \sum_{k \in \mathbb{Z}_{+}}(-1)^{k(r+1)}(2 k+1)^{-r-1}, \quad r \in \mathbb{Z}_{+},
$$

- так называемые константы Фавара. Равенство достигается на эйлеровом перфектном сплайне

$$
x_{n}(t)=\frac{4}{\pi} \sum_{k \in \mathbb{Z}_{+}} \frac{\sin ((2 k+1) t-n \pi / 2)}{(2 k+1)^{n+1}}
$$

$\left(x_{n}^{(n)}(t)=\operatorname{sgn} \sin t\right)$.

Естественная формализация задачи о нахождении точной константы в неравенстве Колмогорова приводит к задаче оптимального управления с фазовьми ограничениями, причем на всей прямой. Непосредственному ее рассмотрению мы предпошлем решение одной частной задачи на $\mathbb{R}_{+}$(задачи Ландау-Арестова), на примере которой, с одной стороны, легко понять основные моменты исследования в обшей постановке, а с другой стороны, она является примером "частного" решения, которьм (как уже говорилось) предполагается посвятить отдельную публикацию. Так что эта задача послужит в нее как бы введением.

Итак, мы будем решать следуюшую экстремальную задачу:

$$
\|\dot{x}(\cdot)\|_{C^{b}\left(\mathbb{R}_{+}\right)} \rightarrow \max , \quad\|x(\cdot)\|_{C^{b}\left(\mathbb{R}_{+}\right)} \leqslant \delta, \quad\|\ddot{x}(\cdot)\|_{L_{r}\left(\mathbb{R}_{+}\right)} \leqslant 1,
$$

где числа $\delta$ и 1 выбраны из тех же соображений, что и раньше.

Решение. 1) Формализация. Рассмотрим задачу

$$
\begin{aligned}
& -x_{2}(0) \rightarrow \min , \\
& \left|x_{1}(t)\right| \leqslant \delta \forall t \in \mathbb{R}_{+}, \quad \int_{\mathbb{R}_{+}}|u(t)|^{r} d t \leqslant 1, \quad x=x_{1}, \dot{x}_{1}=x_{2}, \dot{x}_{2}=u .
\end{aligned}
$$

Если $r=\infty$, то последнее неравенство в (ii) заменяется на неравенство: $|u(t)| \leqslant 1$ для п.в. $t \in \mathbb{R}_{+}$.

2) Принцип Лагранжа. Это задача классического вариационного исчисления при $r<\infty$ (оптимального управления при $r=\infty$ ) с фазовыми ограничениями. Структура задачи позволяет сделать следующее предположение о том, как должно вести себя "разумное" ее решение $\widehat{x}(\cdot)$ : ясно, что $\dot{\widehat{x}}(0)>0$ и поэтому $\widehat{x}(0)=-\delta$. Далее, $\widehat{x}(\cdot)$ растет и достигает верхней границы в некоторой точке $T$, т.е. $\widehat{x}(T)=\delta$, а затем идет по границе, т.е. $\dot{\widehat{x}}(T)=0$ и $\widehat{x}(t)=\delta$ при $t \geqslant T$. Исходя из этих предположений, функция Лагранжа задачи (ii) имеет вид:

$$
\mathscr{L}=-x_{2}(0)-\alpha x_{1}(0)+\beta x_{1}(T)+\lambda\left(\int_{0}^{T}|u|^{r} d t-1\right)+\int_{0}^{T}\left(p_{1}\left(\dot{x}_{1}-x_{2}\right)+p_{2}\left(\dot{x}_{2}-u\right)\right) d t,
$$


где $\alpha, \beta \geqslant 0$ (неотрицательная мера, сосредоточенная в точках 0 и $T$, которая появляется при учете фазовых ограничений) и слагаемое с сомножителем $\lambda$ отсутствует, если $r=\infty$.

3) Исследование. Если $\widehat{x}(\cdot)$ - решение задачи (i), то найдутся такие $\lambda \geqslant 0$, $p=\left(p_{1}, p_{2}\right), \alpha \geqslant 0$ и $\beta \geqslant 0$, что справедливы соотношения (уравнение Эйлера и условия трансверсальности): $-\dot{p}_{1}=0,-\dot{p}_{2}-p_{1}=0, p_{2}=\lambda r|u|^{r-1} \operatorname{sgn} u$ $\left(u=\operatorname{sgn} p_{2}\right.$ при $\left.r=\infty\right)$ и $p_{1}(0)=-\alpha, p_{1}(T)=-\beta, p_{2}(0)=-1, p_{2}(T)=0$. Отсюда сразу следует, что

$$
\begin{gathered}
p_{2}(t)=-1+\frac{t}{T}, \quad \alpha=\beta=\frac{1}{T}, \\
\ddot{\widehat{x}}(t)=-(\lambda r)^{1-r^{\prime}}\left(1-\frac{t}{T}\right)^{r^{\prime}-1} \quad(\ddot{\widehat{x}}(t)=-1 \text { при } r=\infty) .
\end{gathered}
$$

Поскольку $\widehat{x}(T)=\delta$ и $\dot{\widehat{x}}(T)=0$, то из (а) вытекает, что

$$
\begin{gathered}
x(t)=\delta-\left(\frac{(\lambda r)^{1-r^{\prime}} T^{2}}{r^{\prime}\left(r^{\prime}+1\right)}\right)\left(1-\frac{t}{T}\right)^{r^{\prime}+1} \\
\left(x(t)=-\frac{1}{2}(t-T)^{2}+\delta \text { при } r=\infty\right) .
\end{gathered}
$$

Осталось определить две константы $\lambda$ и $T$ (только $T$, если $r=\infty$ ). Они находятся из предположения: $\widehat{x}(0)=-\delta$ (при $r=\infty$ сразу получаем, что $T=2 \sqrt{\delta}$ ) и условия дополняющей нежесткости

$$
\int_{0}^{T}|\ddot{x}|^{r} d t=1
$$

Подставляя $t=0$ в (b) и (а) в (c), получаем, что $\lambda=r^{-1}\left(T /\left(r^{\prime}+1\right)\right)^{1 / r^{\prime}}$, $T=T(r, \delta)=\left(2 r^{\prime}\left(r^{\prime}+1\right)^{1 / r^{\prime}}\right)^{r^{\prime} /\left(r^{\prime}+1\right)} \delta^{r^{\prime} /\left(r^{\prime}+1\right)}$. Отсюда следует, что $\widehat{x}(t)=\delta-$ $2 \delta(1-t / T(r, \delta))^{r^{\prime}+1}$, если $0 \leqslant t \leqslant T(r, \delta)$, и $\widehat{x}(t)=\delta$, если $t \geqslant T(r, \delta)$. Отметим, что соотношения для $T$ и $\widehat{x}(\cdot)$ охватывают и случай $r=\infty$.

Из условия минимума функции Лагранжа в экстремальной точке следует равенство для всех функций $x(\cdot) \in \mathscr{W}_{\infty, r}^{2}\left(\mathbb{R}_{+}\right)$(непосредственная его проверка тривиальна):

$$
\dot{x}(0)=\frac{x(T)-x(0)}{T}+\int_{0}^{T} p(t) \ddot{x}(t) d t,
$$

где $T=T(r, \delta)$ и $p(t)=p_{2}(t)=t / T-1$. Используя это тождество, решим (как и раньше) три задачи (введя предварительно обозначение: $K(r)=2^{1 /\left(r^{\prime}+1\right)} \times$ $\left.\left(\left(r^{\prime}+1\right) / r^{\prime}\right)^{r^{\prime} /\left(r^{\prime}+1\right)}\right)$.

А) Неравенство для производных. Оценивая в (iii) первое слагаемое по модулю, а второе по неравенству Гёльдера, получаем, что для любой допустимой в (ii) функции $x(\cdot) \in \mathscr{W}_{\infty, r}^{2}\left(\mathbb{R}_{+}\right)$справедливо неравенство

$$
|\dot{x}(0)| \leqslant \frac{2 \delta}{T}+\left(\frac{T}{r^{\prime}+1}\right)^{1 / r^{\prime}}=K(r) \delta^{1 /\left(r^{\prime}+1\right)} .
$$


Это неравенство точное, так как на функции $\widehat{x}(\cdot)$ оно обрашается в равенство. Отсюда следует, что $\widehat{x}(\cdot)$ - решение задачи (ii) и, тем самым, задачи (i). Отсюда же и из элементарных соображений однородности вытекает, что для всех функций $x(\cdot) \in \mathscr{W}_{\infty, r}^{2}\left(\mathbb{R}_{+}\right)$вьполняется точное неравенство

$$
\|\dot{x}(\cdot)\|_{C^{b}\left(\mathbb{R}_{+}\right)} \leqslant K(r)\|x(\cdot)\|_{C^{b}\left(\mathbb{R}_{+}\right)}^{1 /\left(r^{\prime}+1\right)}\|\ddot{x}(\cdot)\|_{L_{r}\left(\mathbb{R}_{+}\right)}^{r^{\prime} /\left(r^{\prime}+1\right)}
$$

т.е. $K_{\mathbb{R}_{+}}(2,1, \infty, \infty, r)=K(r)$.

В) Двойственная задача к (ii) (см. задачи (А) и (A*)) есть задача оптимального восстановления функционала $x(\cdot) \mapsto \dot{x}(0)$ на классе

$$
W_{\infty, r}^{2}\left(\mathbb{R}_{+}\right)=\left\{x(\cdot) \in \mathscr{W}_{\infty, r}^{2}\left(\mathbb{R}_{+}\right):\|\ddot{x}(\cdot)\|_{L_{r}\left(\mathbb{R}_{+}\right)} \leqslant 1\right\}
$$

при условии, что функция $x(\cdot)$ в метрике $C^{b}\left(\mathbb{R}_{+}\right)$известна с точностью до $\delta$. Функционал $y^{*}$ на $C^{b}\left(\mathbb{R}_{+}\right)$, который сопоставляет $y(\cdot) \in C^{b}\left(\mathbb{R}_{+}\right)$величину, равную первому слагаемому в (iii) (с заменой $x(\cdot)$ на $y(\cdot)$ ), и есть оптимальный метод восстановления. Действительно, обозначая $\xi(\cdot)=x(\cdot)-y(\cdot)$ и поступая точно так же, как при решении соответствуюшей задачи в случае Габушина (но используя те же оценки для интегралов, что и выше), приходим к соотношению

$$
\dot{x}(0)-\left\langle y^{*}, y(\cdot)\right\rangle \leqslant K(r) \delta^{1 /\left(r^{\prime}+1\right)},
$$

из которого по тем же соображениям, что и раньше, следует

$$
\dot{x}(0) \approx \frac{y(T)-y(0)}{T},
$$

и при этом

$$
e\left(\dot{x}(0), W_{\infty, r}^{2}\left(\mathbb{R}_{+}\right), \delta\right)=K(r) \delta^{1 /\left(r^{\prime}+1\right)} .
$$

С) Пусть $N>0$. Тогда если $\delta>0$ таково, что $T=T(r, \delta)=2 / N$, то норма функционала $x^{*}$, сопоставляющего $x(\cdot) \in C^{b}\left(\mathbb{R}_{+}\right)$первое слагаемое в (iii), равна $N$. Используя (iii) для оценки сверху величины (C) и (D) для ее оценки снизу, получаем, что

$$
E\left(N, \dot{x}(0), W_{\infty, r}^{2}\left(\mathbb{R}_{+}\right)\right)=\left(\frac{2}{N\left(r^{\prime}+1\right)}\right)^{1 / r^{\prime}}
$$

и, тем самым, $x^{*}$ - наилучший приближающий функционал в задаче Стечкина.

Перейдем непосредственно к доказательству неравенства Колмогорова. Содержательная его часть - в доказательстве соответствуюшего неравенства на окружности $\mathbb{T}$. Итак, на пространстве

$$
\mathscr{W}_{\infty}^{n}(\mathbb{T})=\left\{x(\cdot) \in C(\mathbb{T}): x^{(n-1)}(\cdot) \text { абсолютно непрерывна, } x^{(n)}(\cdot) \in L_{\infty}(\mathbb{T})\right\}
$$

будем изучать следуюшую экстремальную задачу:

$$
\left\|x^{(k)}(\cdot)\right\|_{C(\mathbb{T})} \rightarrow \max , \quad\|x(\cdot)\|_{C(\mathbb{T})} \leqslant \delta, \quad\left\|x^{(n)}(\cdot)\right\|_{L_{\infty}(\mathbb{T})} \leqslant 1 .
$$


РЕшЕниЕ. 1) Формализация. В качестве таковой рассматриваем задачу

$$
\begin{gathered}
-x_{k+1}(0) \rightarrow \min , \\
\max _{-\pi \leqslant t \leqslant \pi}\left|x_{1}(t)\right| \leqslant \delta, \quad|u(t)| \leqslant 1, \quad x=x_{1}, \dot{x}_{1}=x_{2}, \ldots, x^{(n)}=u,
\end{gathered}
$$

где первое неравенство в (ii) понимается для всех, а второе - для п.в. $t \in \mathbb{T}$.

2) Принцип Лагранжа. Задача (ii) - задача оптимального управления с фазовьми ограничениями. Ее функция Лагранжа имеет вид:

$$
\begin{aligned}
\mathscr{L}=-\int_{\mathbb{T}} & x_{k+1}(t) \delta(t) d t+\int_{\mathbb{T}}\left(\left|x_{1}(t)\right|-\delta\right) d \mu(t) \\
& \quad+\int_{\mathbb{T}}\left(\sum_{i=1}^{n-1} p_{i}\left(\dot{x}_{i}-x_{i-1}\right)+p_{n}\left(\dot{x}_{n}-u\right)\right) d t+\sum_{s=1}^{n} \nu_{s}\left(x_{s}(-\pi)-x_{s}(\pi)\right),
\end{aligned}
$$

где $\delta(\cdot)-\delta$-функция (мы формально записываем минимизируемый функционал в виде интеграла), а последняя сумма учитьвает периодичность функций.

3) Исследование. Мы будем исходить из предположения (как и Колмогоров, соответствуюшая гипотеза была высказана Г.Е. Шиловым), что решением (ii) является эйлеров перфектный сплайн $x_{m n}(\cdot)$ для некоторого $m \in \mathbb{N}$ (или его сдвиг). Сплайн однозначно определяется условием $x_{m n}^{(n)}(t)=\operatorname{sgn} \sin m t$. Поскольку $\left\|x_{m n}(\cdot)\right\|_{C(\mathbb{T})}=K_{n} / m^{n}$, то мы должны считать, что $\delta$ не меншше этой величины. Далее для определенности будем предполагать, что $k=1$, а $n$ кратно четырем. В этом случае сплайн $x_{m n}(\cdot)$ не нужно сдвигать (максимум первой производной достигается в нуле), и так как его экстремумы находятся в точках $\pi / 2 m+s \pi / m$, $-m \leqslant s \leqslant m-1$, то в этих же точках должна быть сосредоточена мера $d \mu(t)$.

Функция $\mathscr{L}$ достигает своего минимума в экстремальной точке, т.е.

$$
\mathscr{L}\left(x_{1}(\cdot), \ldots, x_{n}(\cdot), u(\cdot)\right) \geqslant \mathscr{L}\left(\widehat{x}_{1}(\cdot), \ldots, \widehat{x}_{n}(\cdot), \widehat{u}(\cdot)\right) .
$$

Полагая в этом неравенстве $x_{1}(\cdot)=x(\cdot), x_{2}(\cdot)=\dot{x}(\cdot), \ldots, x_{n}(\cdot)=x^{(n-1)}(\cdot)$ и $u(\cdot)=0$, приходим к равенству

$$
\dot{x}(0)=\sum_{s=-m}^{m-1} \mu_{s} x\left(\frac{\pi}{2 m}+\frac{s \pi}{m}\right)+\int_{\mathbb{T}} p_{n}(t) x^{(n)}(t) d t,
$$

которое при некоторых $\mu_{s},-m \leqslant s \leqslant m-1$, и $p_{n}(\cdot)$ должно вьполняться для всех $x(\cdot)$.

Подставим в это тождество вместо $x(\cdot)$ ядро Бернулли $\varphi_{n}(\cdot-\tau)\left(\varphi_{n}^{(n)}(\cdot)=\right.$ $\delta(\cdot)-1 / 2 \pi), \tau \in \mathbb{T}$. Тогда получим

$$
p_{n}(\tau)=\varphi_{n-1}(-\tau)-\sum_{s=-m}^{m-1} \mu_{s} \varphi_{n}\left(\frac{\pi}{2 m}+\frac{s \pi}{m}-\tau\right)+\frac{1}{2 \pi} \int_{\mathbb{T}} p_{n}(\tau) d \tau .
$$

Положим теперь $x_{1}(\cdot)=\cdots=x_{n}(\cdot)=0$ в (а). Тогда получим неравенство, из которого сразу следует, что $\widehat{u}(t)(=\operatorname{sgn} \sin m t)=\operatorname{sgn} p_{n}(t)$. Значит, числа $\mu_{s}, s=-m, \ldots, m-1$, должны быть прежде всего "потрачены" на то, чтобы 
функция справа в (iv) имела нули в нулях функции $t \mapsto \sin m t$, т.е. в точках $s \pi / m$. Из теоремы Ролля вытекает, что это возможно, и при этом перемены знака этой функции совпадают с переменами знака $\sin m \cdot$, а знаки множителей $\mu_{s}$ согласованы со знаками перфектного сплайна. Отсюда (как и раньше), используя (iii), получаем точную оценку для модуля соответствуюшей производной в нуле, и поэтому $x_{m n}(\cdot)$ - решение задачи (ii), а значит, и задачи (i).

Тождество (iii) дает также решение задачи об оптимальном восстановлении функционала $x(\cdot) \mapsto x^{(k)}(0)$ и решение задачи Стечкина.

Переход от задачи (i) к соответствуюшей задаче на прямой осушествляется стандартньм образом (см., например, [26]).

В работе [5], опубликованной в 1957 году, Стейн показал, что константа $K_{\mathbb{R}}(n, k, 1,1,1)$ совпадает с константой в неравенстве Колмогорова. Это единственный случай в нашей коллекции, где теория экстремальных задач пасует.

Отметим, что за неимением места мы не смогли включить в эту статью другие интересные обобшения рассмотренных в первой части задач (например, задачи Тайкова и Колмогорова на сфере и др.). Результаты по задаче Тайкова на сфере (в постановке несколько отличной от принятой здесь) докладывались А. А. Ильиньм на семинаре В. М. Тихомирова, и в ближайшее время они будут опубликованы.

ЗАКЛЮчЕнИЕ. Если посмотреть на всю совокупность когда бы то ни было точно решенных экстремальных задач, то обнаружится, что подавляющее большинство из них допускает "стандартное" решение по типу тех, которые приведены в данной статье. Тем более стоит отметить задачи, которые не допускают таких решений. Важнейший класс таких задач - многомерные. Как решать задачу (квадратичную по своей природе!) типа Тайкова, Любича-Купцова в четверти плоскости? Мы не говорим уже о многомерных задачах оптимального управления, обобщающих колмогоровский случай.

\section{Список литературы}

1. Landau E. Ungleichungen für zweimal differenzierbar Fumktiontn // Proc. London. Math. Soc. 1913. V. 2. № 13. P. 43-49.

2. Hadamard J. Sur le module maximumd'une fonction et de ses derivees // C. R. Sci. 1914. V. 41. P. $68-72$.

3. Колмогоров А. Н. О неравенствах между верхними гранями последовательных производных произвольной функции на бесконечном интервале // Избранные труды. Математика и механика. М.: Наука, 1985. С. 252-261.

4. Харди Г. Г., Литтлвуд Дж. Е., Полиа Г. Неравенства. М.: ИЛ, 1948.

5. Stein E. M. Functions of exponential type // Ann. of Math. (2). 1957. V. 65. №3. P. $582-592$.

6. Тайков Л. Н. Неравенства типа Колмогорова и наилучшие формулы численного диффференцирования // Матем. заметки. 1968. Т. 4. № 2. С. 233-238.

7. Любич Ю. И. О неравенствах между степенями линейного оператора // Изв. АН СССР. Сер. матем. 1960. Т. 24. С. 825-864.

8. Купцов Н. П. Колмогоровские оценки для производных в $L_{2}(0, \infty) / /$ Труды МИАН. 1975. T. 138. C. $94-117$.

9. Габушин В. Н. О наилучшем приближении оператора дифференцирования на полупрямой // Матем. заметки. 1969. Т. 6. № 5. С. 573-582. 
10. Тихомиров В. М., Магарил-Ильяев Г. Г. Неравенства для производных // Избранные труды. Математика и механика. М.: Наука, 1985. С. 386-390.

11. Арестов В. В. Приближение неограниченных операторов ограниченньми и родственные экстремальные задачи // УМН. 1996. Т. 51. №6. С. 89-124.

12. Иоффе А. Д., Тихомиров В. М. Теория экстремальных задач. М.: Наука, 1974.

13. Тихомиров В. М. Некоторые вопросы теории приближений. М.: Изд-во МГУ, 1976.

14. Алексеев B. М., Тихомиров В. М., Фомин С. В. Оптимальное управление. М.: Наука, 1979.

15. Алексеев В. М., Галеев Э. М., Тихомиров В. М. Сборник задач по оптимизации. М.: Наука, 1984.

16. Tikhomirov V. M. Fundamental Principles of the Theory of Extremal Problems. Chichester: J. Wiley \& Sons, 1986.

17. Галеев Э. М., Тихомиров В. М. Краткий курс теории экстремалњњых задач. М.: Наука, 1989.

18. Смоляк C. А. Об оптимальном восстановлении функций и функционалов от них // Дис. ... канд. физ.-матем. наук. М., 1965.

19. Магарил-Ильяев Г. Г., Осипенко К. Ю. Об оптимальном восстановлении функционалов по неточным данным // Матем. заметки. 1991. Т. 50. №1. С. 85-93.

20. Буслаев A. П., Тихомиров $B$. . О неравенствах для производных в многомерном случае // Матем. заметки. 1979. Т. 25. №1. С. 59-74.

21. Виленкин H. Я. Специальные функции и теория представлений групп. М.: Наука, 1991.

22. Динь Зунг, Тихомиров В. М. О неравенствах для производных в метрике $L_{2} / /$ Вестн. МГУ. Сер. 1. Матем., мех. 1979. № 2. С. 7-11.

23. Зигмунд А. Тригонометрические ряды. Т. I, II. М.: Мир, 1965.

24. Магарил-Ильяев Г. Г., Тихомиров В. М. О неравенстве Колмогорова для дробных производных на полупрямой // Anal. Math. 1981. Т. 7. № 1. С. 37-47.

22. Градитейн И. С., Рыжик И. М. Таблицы интегралов, сумм и произведений. М.: Физматлит, 1962.

26. Cavaretta A.S. An elementary proof of Kolmogorov's theorem // Amer. Math. Monthly. 1974. V. 81. № 5. P. 480-486. 